\title{
MOSSES OF SALAIR-KUZNETSK REGION (ALTAI-SAYAN MOUNTAIN COUNTRY) AND ADJACENT PLAINS OF WEST SIBERIA
}

\author{
МХИ САЛАИРО-КУЗНЕЦКОГО РЕГИОНА (АЛТАЕ-САЯНСКАЯ ГОРНАЯ ОБЛАСТЬ) И \\ ПРИЛЕГАЮЩИХ ТЕРРИТОРИЙ ЗАПАДНО-СИБИРСКОЙ РАВНИНЫ \\ OLGA YU. PISARENKO ${ }^{1}$ \\ ОЛЬГА Ю. ПИСАРЕНКО ${ }^{1}$
}

Abstract

\begin{abstract}
The paper summarizes the data of long-term bryological explorations in south-eastern part of West Siberia - in territories at the junction of the West Siberian plain and adjacent north-western part of Altai-Sayan Mountain region. The list includes 425 moss species of 49 families and 160 genera. Taxa are characterized by their occurrence in 9 phytogeographic subdivisions, as well as by the altigudinal range. Annotations and bibliography are included. Moss flora of individual regions and species distribution within the area are discussed.
\end{abstract}

Резюме

Обобщены данные многолетних бриологических исследований в юго-западной части Сибири - на территориях на стыке Западно-Сибирской равнины и Алтае-Саянской горной страны. Список мхов включает 425 видов из 49 семейств и 160 родов; указаны распределение и встречаемость видов по 9 фитогеографическим регионам территории; по отдельным видам даны комментарии. Обсуждаются особенности бриофлоры рассматриваемых регионов и закономерности распределения видов. Приводится библиография.

KEYWORDS: brophytes, flora, phytogeography, West Siberian Plain, Salair Ridge, Kuznetsky Alatau Range, Kulunda Steppe, Baraba Steppe, Vasyugan Plain

\section{INTRODUCTION}

The study area lies in the center of Eurasia, where the West Siberian plain transits to the Altai-Sayan mountain region. The West Siberian plane is the largest lowland of the Earth, extending up to $2500 \mathrm{~km}$ from north to south and $1900 \mathrm{~km}$ from west to east. Its surface is formed by a thick cover of loose Quaternary sediments. Bioclimatic zones from northern tundra through taiga forest to steppe are well-expressed on the plain due to relatively homogeneous areas. The Altay-Sayan mountain region is the main part of the South Siberia mountain belt (Zyat'kova, 1977), being $1500 \mathrm{~km}$ long and joining the Baikal Mountains in the east. The boundary between the West Siberian plane and the Altai-Sayan mountain country is rather sharp. Neighboring areas of these two physiographic countries provide a convenient plot to find out specific distribution patterns, correlating the latitudinally arranged physiographic zones and the altitudinal belts in mountains.

The results of the author's long-term bryological investigations in this area are summarized. Nomenclature of vascular plants follows Cherepanov (1995), mosses Ignatov et al. (2006) with some latest additions.

\section{STUDY AREA}

The territory under discussion stretches for ober 800 $\mathrm{km}$ from west to east and for about $600 \mathrm{~km}$ from north to south. Bioclimatic zones from south taiga to steppe follow the latitudee. In mountains, the altitudinal belts are well expressed; vegetation changes from mesophytic Betula- and Pinus-forests to Abies-forests and then to highmountain tall-herb communities and finally to mountain tundra. According to the administrative subdivision, the study area belongs to Altai Territory (=Krai) and to Novosibirsk, Kemerovo and partly Tomsk Provinces (Figs. 1-2). Physiograpically the area is divided into nine geographic regions (Fig. 3 \& Table 1).

My investigations in this territory started in 1992. At that time the major part of the territory remained out of the spotlight of bryologists and the catalogue of bryophytes of West Siberia written by Krylov (1925) was the main source of information. Krylov (1.c.) listed localities of 130 species in the plain part of the former Tomsk Province (at that time broader than our whole study area). Attention of few later researchers was focused on mountain regions (Vasiljev, 1974, 1975, 1978; Gudoshnikov, 1976, 1978, 1986) and on huge mire areas in the

\footnotetext{
1 - Central Siberian Botanical Garden, Zolotodolinskaya 101, Novosibirsk 630090 Russia - Россия 630090 Новосибирск, Золотодолинская, 101, Центральный Сибирский ботанический сад СО РАН
} 
Table 1. Regions of study area, theirs abbreviations (cf. Fig.2), main sources of regional bryoflora information. (Not all author's material has been published earlier; collecting period for a region is listed in the end of cells). - Табл. 1. Регионы изучаемой территории и используемые в тексте сокращения; основные источники информации по флорам мхов. (Не все материалы автора были ранее опубликованы; годы сборов указаны внизу ячеек).

Abbreviation Region References on bryofloristic publications and the author collections

Аббревиатура Регион Публикации по бриофлоре и сборы автора

Salair-Kuznetsk region (north-west part of Altai-Sayan mountain country)

KA Kuznetskiy Alatau / Кузнецкий Алатау Vasiljev, 1973, 1974, 1975, 1976, 1978, 1995; Muldiyarov, Lapshina, 1996; Volkova, Muldiyarov, 2000; Pisarenko, 2004, 2007a, 2009a, 2009b; Pisarenko, Nozinkov, 2007; Ignatova, Pisarenko, 2013a; author collections 1992, 1994, 1998, 2000-2005

SM Shoria Mountains / Горная Шория Vasiljev, 1973, 1974, 1975, 1976, 1978, 1995; Gudoshnikov, 1976, 1986; Pisarenko, Nozinkov, 2007; Nozinkov, Pisarenko, 2008; Pisarenko, 2007a, 2012b; Ignatova, Pisarenko, 2013a; author collections 2007, 2010, 2011

SR Salair ridge / Салаирский кряж Gudoshnikov, 1986; Lashchinsky, Pisarenko, 1999; Pisarenko, Lashchinsky, 1999; Pisarenko, 1999, 2007a, 2007b, 2012b; Pisarenko, Nozinkov, 2007; Ignatova, Pisarenko, 2013b; author collections 1992-1996

KD Kuznetskaya depression Pisarenko, Nozinkov, 2007; Pisarenko, 2007a, 2011; author collections 2007-2011

West Siberian plain, south-east coner

/ Кузнецкая котловина

TR Transitional region / Колывань-Томская Logutenko, 1963; Lapshina, Muldiyarov, 1998; Muldiyarov, Chernova, 2002; дуга и Приаргинская равнина Pisarenko, 2007a, 2012c, 2013; Pisarenko, Nozinkov, 2007; Lashchinsky, Pisarenko, 2010; Dyachenko, Taran, 2012b; Pisarenko, Valutskiy, 2013; author collections 2005, 2007-2011

UO upper $\mathrm{Ob} /$ Верхнее Приобье Nozinkov, 2006; Pisarenko, Nozinkov, Zolotov, 2007, 2008; Nozinkov, Taran, 2008; Pisarenko, 2012a, 2012c; Dyachenko, Taran, 2012a; collections 2007-2013

KS Kulunda Steppe / Кулунда Pisarenko, Ignatova, Ignatov, 2001; Nozinkov, 2006; Pisarenko, 2006; Pisarenko, Nozinkov, Zolotov, 2008; author collections 1995, 2003, 2010-2011

BS Baraba Steppe / Бараба

VP Vasyugan plain and adjacent areas of Novosibirsk and Tomsk Provinces / Logutenko, 1963; Valutskiy, 2011; author collections 2009, 2012 Lapshina, Muldiyarov, 1998; Pisarenko, Lapshina, Muldiyarov, 2011; Pisarenko, 2012c; author collections 1998, 2005, 2012, 2013

Васюганье и прилегающие территории

Новосибирской и Томской областей

northern part of the territory (Logutenko, 1963; L'vov, 1973; Muldiyarov, 1979, 1990; Lapshina \& Muldiyarov, 1998). Full bibliography is given in Table 1 . Years of the author's collections are shown ibid.

The north-western part of the Altai-Sayan mountain region is recognized as Salair-Kuznetsk region (Olyunin, 1975). It is a Late Mesozoic-Coenozoic dome-shaped elevation with a lowering in its central part. It is subdivided into Kuznetskiy Alatau, Mountain (Gornaya) Shoria, low Salair ridge and Kuznetskaya depression (Fig. 3).

Kuznetskiy Alatau is stretched meridionally within 53-56 $\mathrm{N}$ and $86-91^{\circ}$ E. Macro-slopes of Kuznetskiy Alatau are asymmetric. The western one is faced to the Kuznetskaya depression; it is steep, formed with a system of large ledges. The eastern macro-slope is gently descending to the Minusinskaya depression. Two macro-slopes differ contrastingly in precipitation and consequently in vegetation and relief. In climatology, this ridge is an important climatic frontier, separating continental and sharply continental climatic sectors (Myachkova, 1983). In phytogeography, this watershed is considered as a part of a boundary between the region of dark coniferous forest of East Europe and West-Siberia (to the west from the ridge), and the region of Larix-forests of East Siberia (to the east) (Lavrenko, 1978). Also Kuznetskiy Alatau is a phytogeographical boundary for some higher rank units of meadow and steppe vegetation (Lavrenko et al., 1991; Makunina \& Maltseva, 2008). The present paper deals only with the western macro-slope of Kuznetsky Alatau.

Detailed characteristics of the region is given by Pisarenko (2004). Most important features for the moss distribution are the following. Altitudes range from 300$500 \mathrm{~m}$ in the northern part to $2000 \mathrm{~m}$ in the south. Elevation intervals from the valleys level to the highest peaks are about $600-700 \mathrm{~m}$. The relief has a tier structure with 2-3 surfaces of peneplainization (Kashmenskaya et al.,1969). Flat watersheds and slightly inclined surfaces are covered with Quaternary sediments. Transition to the upper surface is characterized by a sharp increasing in slope steepness; talus and rock fields are common there. Most widespread rocks are gabbros, granodiorites, granites, gneisses, serpentinites, clayish and crystalline slates. Limestone outcrops are rare and revealed in valleys of only some rivers.

There are no meteorological stations in the central parts of the mountain formations - neither Kuznetskiy Alatau nor Mountain Shoria or Salair Ridge; for these territories there are only scattered episodic data. Few stations are located on mountain foothills where the climate is warmer and drier than in the inner elevated parts. Standard climate diagrams for the most convenient sta- 
tions for every described region are in Fig.4, locations of the stations are in Fig.3.

In Kuznetskiy Alatau and Mountain Shoria mean annual precipitation is several times higher than in neighbor regions and varies from 900-1000 up to 3000-3500 mm according to different data (Pilnikova, 1993; Shpin“, 1980) and different parts. An average annual temperature is about $0{ }^{\circ} \mathrm{C}$. Winter snow depth varies from $20-40$ $\mathrm{cm}$ on flat mountain tops to $170-190 \mathrm{~cm}$ in the subalpine belt. When thickness of snow cover exceeds $150 \mathrm{~cm}$, it keeps soil non-freezing during the winter period, temperatures on a soil surface being about $0^{\circ} \mathrm{C}$ (Latchinsky $\&$ Demidenko, 2005). Snow spots may persist for the whole summertime on northern slopes near ridge tops.

In vegetation of the territory, there are 3 mountain belts: a forest-belt, a tall-herbaceous belt, and a tundrabelt. Forests of Abies sibirica with a rich herbs layer occupy slopes of all expositions from river valleys up to $1100 \mathrm{~m}$. At 1100-1250 m a.s.l. tall-herbaceous communities prevail. High-mountain tundra is presented as small isolated areas like islands on flat tops of the upper surface of peneplainization. Smooth forms of relief, huge amount of precipitation and cold climate promote mires development. Mires are diverse and represented in all mountain belts.

Vegetation of the area has been described in a number of publications (Kuminova, 1950; Sedelnikov, 1979; Latchinsky, 1996; Lapshina \& Muldiyarov, 1995; Muldiyarov \& Lapshina, 2000; and others), and briefly overviewed also by Pisarenko (2004).

Mountain (Gornaya) Shoria is situated southward from the Tom River and differs from Kuznetskiy Alatau by smoother relief with elevation intervals between valleys and ridges being 200-300 m. The highest peaks are Patyn Mt. (1628 m) and Pustag Mt. (1570 m), but most peaks are lower than $1200 \mathrm{~m}$ and do not exceed the tree line. Mean annual precipitation and temperature vary from $1300-2000 \mathrm{~mm}$ and $-1,5^{\circ} \mathrm{C}$ in central highmountain parts to $800 \mathrm{~mm}$ and $+1,2^{\circ} \mathrm{C}$ in periphery.

The prevailing type of primeval vegetation here is chernevaia taiga, the most thermophilic and humid type of forest in South Siberia (Polikarpov et al., 1986). It occurs also at the lower elevations in Kuznetskiy Alatau and on the western macroslope of the Salair Ridge. The peculiar composition and structure of chernevaya taiga was discussed by Krylov (1891, 1898), Kuminova (1950), Polozhii \& Krapivkina (1985) Ioschenko \& Laschinsky (1994), Laschinsky (2009). The canopy of Populus tremula and Abies sibirica is open and tree groups form a mozaic with tall-herb meadows. Herb layer is closed and tall; the most abundant are Aconitum septentrionale, Crepis sibirica, Cirsium helenoides, Saussurea latifolia, Euphorbia lutescens. Average height of herbage is 1-1.5 m, but generative sprouts of some species (Alfredia cernua, Delphinium elatum, Heracleum dissectum, Anthriscus sylvestris, and others) often exceed $3 \mathrm{~m}$. Peculiarity of vegetation of Moun-

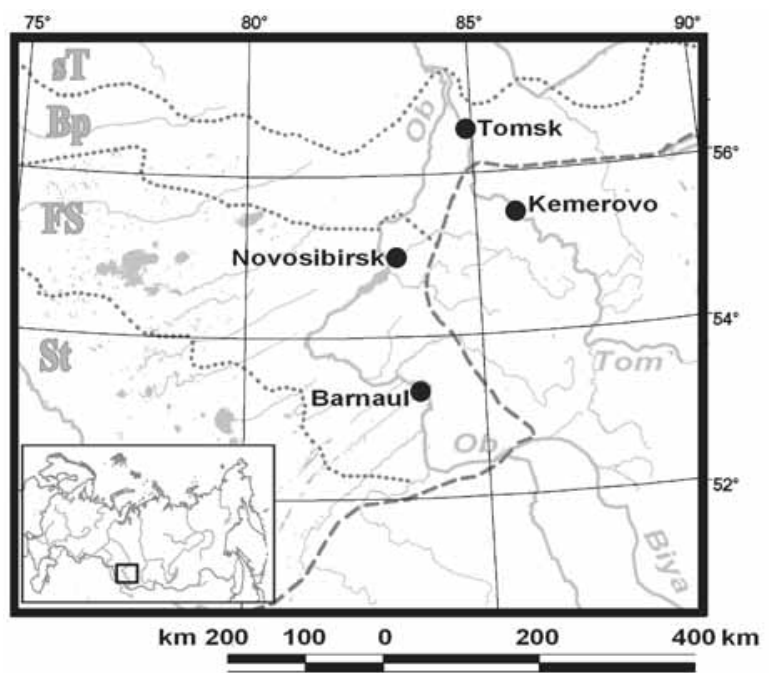

Fig. 1. Study area, showing location of bioclimatic zones. Dotted line marks off West Siberian plane (on the left) and AltaySayan mountain country (on the right) by Gorodetskaya \& Lazukov, 1975; Olyunin 1975. Bioclimatic zones are abbreviated: $\mathbf{s T}$ - south taiga; $\mathbf{B p}$ - subtaiga (native small-lived deciduous forests with Betula pendula dominance); FS - forest-steppe; St - steppe (boundaries - by Il'ina et al., 1985).

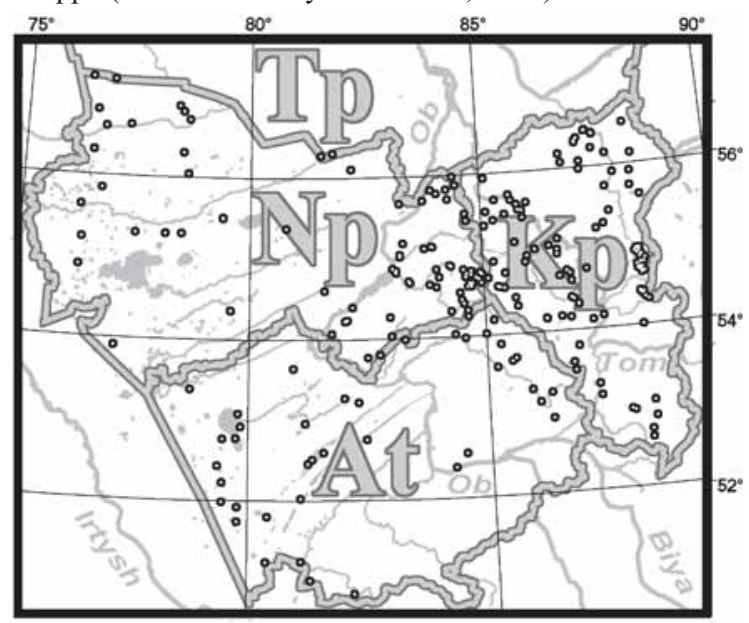

Fig. 2. Studied localities and their distribution in administrative units: At - Altai Territory ; Np - Novosibirsk Province, $\mathbf{K p}$ - Kemerovo Province, Tp - Tomsk Province.

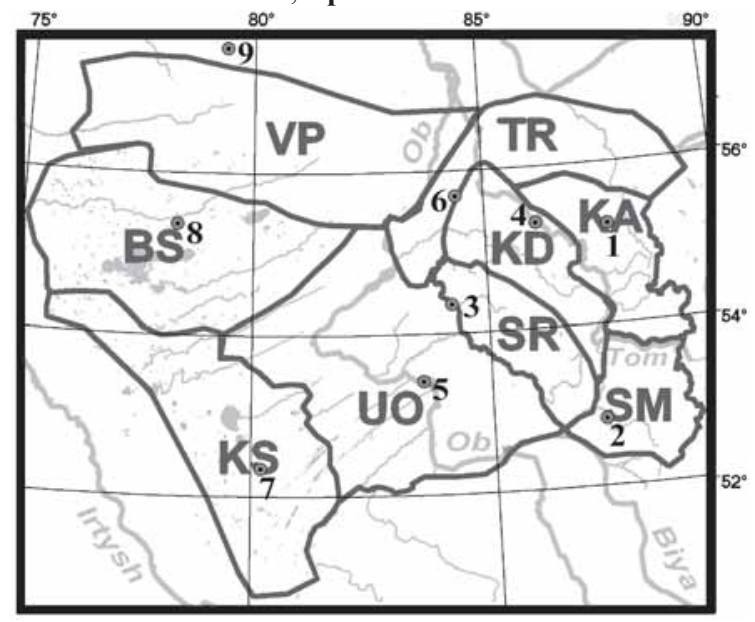

Fig. 3. Geographic regions. Abbreviation are explained in Table 1. Numbers refer to meteorological stations, cf. Fig. 4. 

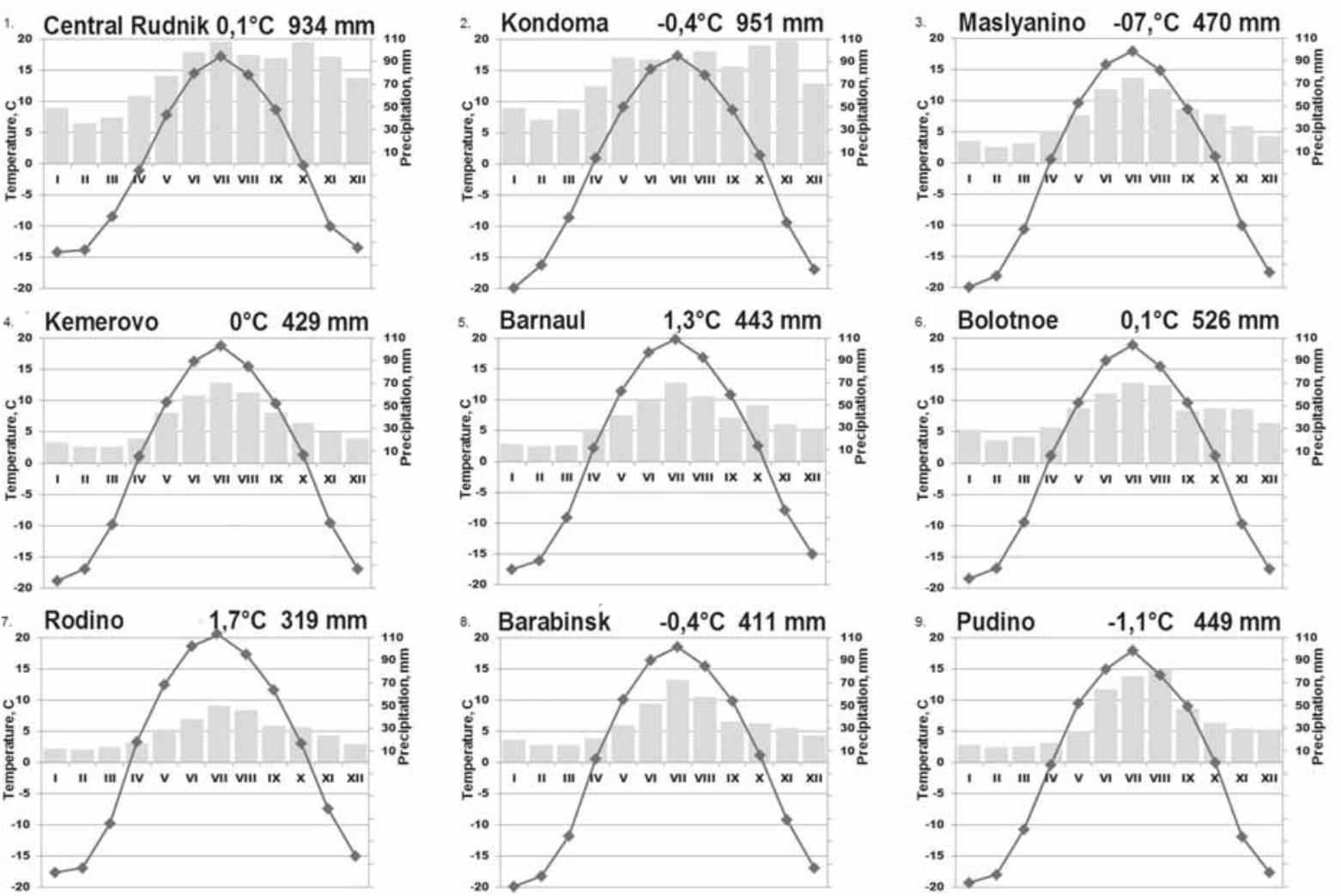

Fig. 4. Climate diagrams for study area according to Pilnikova (1993). The top of each diagram lists the name of the climate station, its average annual temperature $(\mathrm{C})$ and its average annual precipitation $(\mathrm{mm})$. Numbers correspond to those shown in Fig.

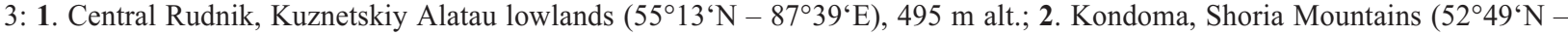
$\left.87^{\circ} 17^{\circ} \mathrm{E}\right), 354 \mathrm{~m}$ alt.; 3. Maslyanino, Salair Ridge foothills $\left(54^{\circ} 20^{\circ} \mathrm{N}-84^{\circ} 13^{\circ} \mathrm{E}\right), 198 \mathrm{~m}$ alt.; 4. Kemerovo, Kuznetskaya depression $\left(55^{\circ} 17^{\circ} \mathrm{N}-86^{\circ} 06^{\circ} \mathrm{E}\right), 260 \mathrm{~m}$ alt.; 5. Barnaul, Upper Ob $\left(53^{\circ} 24^{\circ} \mathrm{N}-83^{\circ} 34^{\circ} \mathrm{E}\right), 157 \mathrm{~m}$ alt.; 6. Bolotnoe, transitional region $\left(55^{\circ} 40^{`} \mathrm{~N}-84^{\circ} 23^{\circ} \mathrm{E}\right), 194 \mathrm{~m}$ alt.; 7. Rodino, Kulunda Stepper $\left(52^{\circ} 30^{`} \mathrm{~N}-80^{\circ} 12^{`} \mathrm{E}\right), 160 \mathrm{~m}$ alt.; 8. Barabinsk, Baraba Stepper

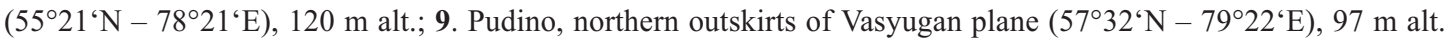

tain Shoria is the Kuzedeevo "Linden island" - a forest massive with abundant Tilia sibirica in the tree layer. In South Siberia, Tilia sibirica is also known from some scattered locations in the Altai, Kuznetskiy Alatau and Salair Ridge; but in Mountain Shoria, the species is rather more common. Kuzedeevo "Linden island" is the biggest from known Tilia-forests; its size is about $18 \times 6$ $\mathrm{km}$. Besides the abundance of Tilia sibirica, the community is characterized by the presence of Asarum europaeum, Festuca altissima, Galium odoratum, Geranium robertianum, Polystichum braunii, which have disjunctive distribution in Siberia and are considered here as Tertiary relicts (Polozhii \& Krapivkina, 1985). Due to anthropogenic pressure, chernevaia taiga is replaced by secondary birch and aspen forests in many areas.

Salair Ridge is a low, slightly incised peneplain with average altitudes ca. $400 \mathrm{~m}$ (Vdovin, 1988). Salair is situated about $100 \mathrm{~km}$ west from Kuznetskiy Alatau and almost parallel to the latter. Macroslopes of Salair are also asymmetric, the eastern one being steep, while the western is more gentle. The ridge surface is covered with a thick layer of quaternary sediments, mainly eolian loess, which is especially thick on the western slope. Rocky outcrops occur mainly in river valleys. Annual precipitation is about $800 \mathrm{~mm}$ on the western slope, declining to $400 \mathrm{~mm}$ in the rain shadow on the eastern one (Lashchinskiy \& Sedelnikov, 1991).

The ridge is totally within the forest belt. Chernevaia taiga completely covers the wet gentle and loamy western macro-slope, while more dry and drained eastern macro-slope has Betula pendula or Pinus sylvestris forests, sometimes with admixture of Larix sibirica. The cover of herb layer is about 60-80\%; Carex macroura, Brachypodium pinnatum, Calamagrostis arundinacea are abundant. Petrophytic steppe with Festuca valesiaca, Koeleria cristata, Potentilla acaulis, Artemisia frigida, Carex duriuscula occurs on steep and sunny faces among forest. Some xerophytic mosses participate in these communities. Mires are rare and small, being mostly of mesotrophic-type. Syntaxonomy of the vegetation is comprehensively described by Lashchinskiy (2009).

Kuznetskaya depression is an elevated hilly plain with altitudes mostly 200-300 m. In the north, the plain has no sharp border; other verges are well-defined in relief. In the south the plain is limited with Mountain Shoria, in the west - with Salair ridge, in the east - with 
Kuznetskiy Alatau. In the center of the eastern part of the plain near Kuznetskiy Alatau foothills, there are specific geological structures, a so-called "Melafir horseshoe" - the basalt mold of Permian age. The highest northern parts of the mold rise above the plain at 200$400 \mathrm{~m}$ and differ from the last in precipitation and vegetation. So, geomorphologically "Melafir horseshoe" belongs to Kuznetskaya depression (Finer, 1969), but in vegetation its northern part is similar to slopes of Kuznetskiy Alatau (Lashchinsky et al., 2011). Mosses of the northern part of "Melafir horseshoe" are included into Kuznetskiy Alatau bryoflora.

Main part of Kuznetskaya depression is within the forest-steppe zone. Zonal vegetation is a complex of small massifs of Betula-forests with steppe meadows and meadow steppes (Kuminova, 1950; Makunina, 1998; Makunina \& Maltseva, 2008). Forests in Kuznetskaya depression are presented mainly by Betula pendula communities. The herb layer of these forests depends on local humidity. Dry habitats are characterized by the dominance of Brachypodium pinnatum, Calamagrostis arundinaceae, Rubus saxatilis sometimes Carex macroura, meadow-steppe herbs such as Peucedanum morissonii, Seseli libanotis, Pulsatilla patens are common. Moist forest types have Populus tremula dominant in the tree canopy; in herb layer Aegopodium podagraria, Aconitum septentrionale, Pteridium aquilinum, Lathyrus gmelinii, Lathyrus vernus, Geranium sylvaticum are common and abundant. Well-developed canopy of tall herbs and meadow grasses (Festuca pratensis, Dactylis glomerata, Elytrigia repens, Phleum pratense, Lupinaster pentaphyllus, Phlomoides tuberosa, Sanguisorba officinalis) is characteristic for the grasslands. The herbadge prevents the development of moss cover. A moss layer takes place only in petrophytic steppes like Salair ones, which occur on most steep and sunny slopes of river valleys or separate hills. Mires here are very rare, they occur only in river valleys, these are swampy tussock-sedge forests with Betula pubescens. In some places along depression sides small rock outcrops are revealed in river valleys.

Plant cover of this region is strongly transformed by agriculture and mining activities: now it is very difficult to find fragments of native vegetation. Periodical ground fires occurring in spring or autumn due to stubble burning on adjoining farmlands are an additional disaster for mosses.

West Siberian plane south-east corner includes regions of the upper Ob, Kulunda, Baraba, Vasyuganie (Vandakurova, 1957; Kuminova et al., 1963; Zarutskaya, 1978; Gorodetskaya \& Lazukov, 1975).

The boundary of the West Siberian plane and SalairKuznetsk region is not well-defined in its relief. Territories on the $\mathrm{Ob}$ right bank in the length from Kamen'-naObi to Tomsk and further to the east along the northern face of Kuznetskiy Alatau are treated by differently in geomorphology (Olyunin, 1975; Gorodetskaya, 1975).
Considering the morphology and vegetation features (Kuminova, 1957; Vandakurova, 1957; Makunina \& Maltseva, 2008), the area is described here as a transitional region. Relief is mild undulated within altitudes $150-300 \mathrm{~m}$. The surface is formed by quaternary sediments, but their layer is not so thick as on most part of West Siberian plane, and small rocky outcrops occur sometimes in river valleys and on hill tops. (In other discussing parts of West Siberia, on the left bank of Ob River, stones are absolutely absent). The region is situated on a boundary of subtaiga and forest-steppe zones. Zonal vegetation is a combination of mesophitic Betula forests and meadows, but the plant cover is strongly transformed by agriculture activity. Steppe communities take place on steep sunny slopes of hills. Mires mainly occur in valleys of small rivers and in flat bottoms of gullies; the most common type is represented by mesotrophic communities with Betula pubescens or willows of Salix cinerea canopy (Kuminova, 1950; Kuminova et al., 1963; Lashchinskiy et al., 2014). On sandy soils along the $\mathrm{Ob}$ River Pinus sylvestris-forests prevail; between massifs of Pinus-forests oligotrophic Sphagnum-bogs occur rarely. In the northern part of the region specific mire massifs were found (Lashchinsky, Pisarenko, 2010): the massifs represent a series of parallel ribbons in river valleys; the central parts of mires are formed by complexes of mesotrophic pools with Carex species and forested peat beds. The periphery of mire systems is often formed by narrow stripes of swampy Picea obovata forests which significantly contribute to biodiversity of the region.

Upper Ob covers plains contouring the north-west face of Altai-Sayan Mountain Country at both sides of the Ob River: Priobskoe plateau and Prisalairskaya plain, Biya-Chumysh upland (Zyat'kova, 1977; Nikolaev, 1988). This region rises above the neighboring western and northern plains by approximately $100 \mathrm{~m}$. The surface is dissected by erosion gullies and ravine systems. Sandy substrates prevail in this area. They form Ob terraces and pave bottoms of ancient ravines of water runoff. On such sort of habitats forests from Pinus sylvestris prevail. The biggest Pinus-forests on the right $\mathrm{Ob}$ River bank are Verhneob (up to $90 \times 50 \mathrm{~km}$ ) and Suzun (up to $70 \times 50 \mathrm{~km}$ ) massifs of forests; on the left bank - Barnaul and Kasmala ribbon forests, their length is $250 \mathrm{~km}$, width - to 3-6 km. In depressions surrounded with Pinus sylvestris-forests, small bog massifs occurs. Poor in nutrients sandy substrates provoke the oligotrophic species appearance (Oxycoccus palustris, Scheuchzeria palustris, Rhynchospora alba, Sphagnum spp.); here they are found far south from the plain part of their areal. Vicinity to windward macroslopes of Altai-Sayan Country has strong influence on the climate of the territory which is expressed primarily in increasing precipitation. Despite a rather southern position, the region is referred to the foreststeppe zone. In zoning schemes, bioclimatic boundaries here are turned in submeridian direction. Zonal vegeta- 

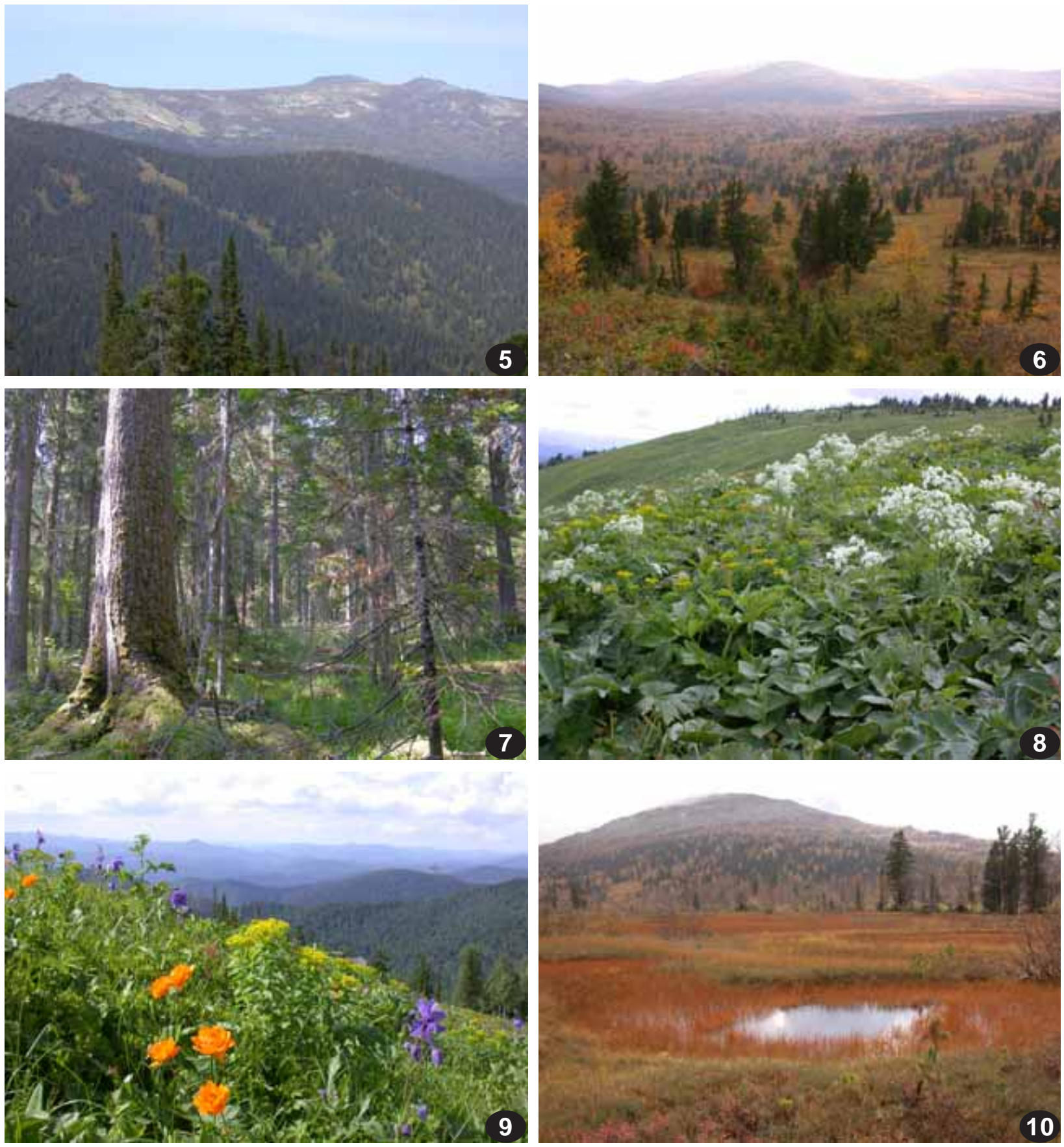

Figs. 5-10. Kuznetskiy Alatau. View on a dominant top; characteristic "goltsy" relief (Figs. 5-6): gentle slopes and flat naked tops covered by tundra and stone fields beneath. Abies sibirica mountain taiga foreground and snow patches beyond, in June (Fig. 5). Beginning of September near timberline $(1100 \mathrm{~m})$ : sparse growth of Pinus sibirica with admixture of undersized forms of Abies sibirica and Betula tortuosa (Fig. 6). Inside of the mountain Abies-forest (Fig. 7): Pinus sibirica, Betula alba s.l., Sorbus sibirica commonly participate in the stand; in herb layer Calamagrostis obtusata, Athyrium distentifolium, Aconitum septentrionale prevail, tall-herb species occur together with taiga umbrophytes Oxalis acetosella, Paris quadrifolia, Gymnocarpium dryopteris etc.; Rhytidiadelphus subpinnatus, Sciuro-hypnum curtum are abundant on soil and it is possible to find Hylocomiastrum umbratum, Sciuro-hypnum ornellanum; from epiphytic species Ulota rehmannii, Iwatsukiella leucotricha, Anacamptodon latidens are most interesting; Pseudoephemerum nitidum once was collected on bare soil on a pass in similar forest. Tall-herbaceous communities with abandant Saussurea latifolia, Euphorbia lutescens, Geranium krylovii, Anthriscus sylvestris (Fig. 8); Trollius asiaticus, Aquilegia glandulosa (Fig. 9) form a belt at 1100-1250 m, where snow is accumulated in winter due to blowing out from tops. On soil and stones under the herb canopy Brachytheciastrum velutinum, Mnium spinosum, Fissidens bryoides, Serpoleskea confervoides, Lescuraea saxicola, Thamnobryum neckeroides, Anomodon rugelii occur. High-mountain mire complex (Fig. 10): small peat ridges (dominants are Betula rotundifolia, Vaccinium uliginosum, Rubus chamaemotus \& Sphagnum fuscum, S. capilllifolium) alternate with watery hollows (dom. Carex limosa, C. lasiocarpa, Scheuchzeria palustris, Sphagnum jensenii, S. papillosum, Warnstorfia fluitans). 

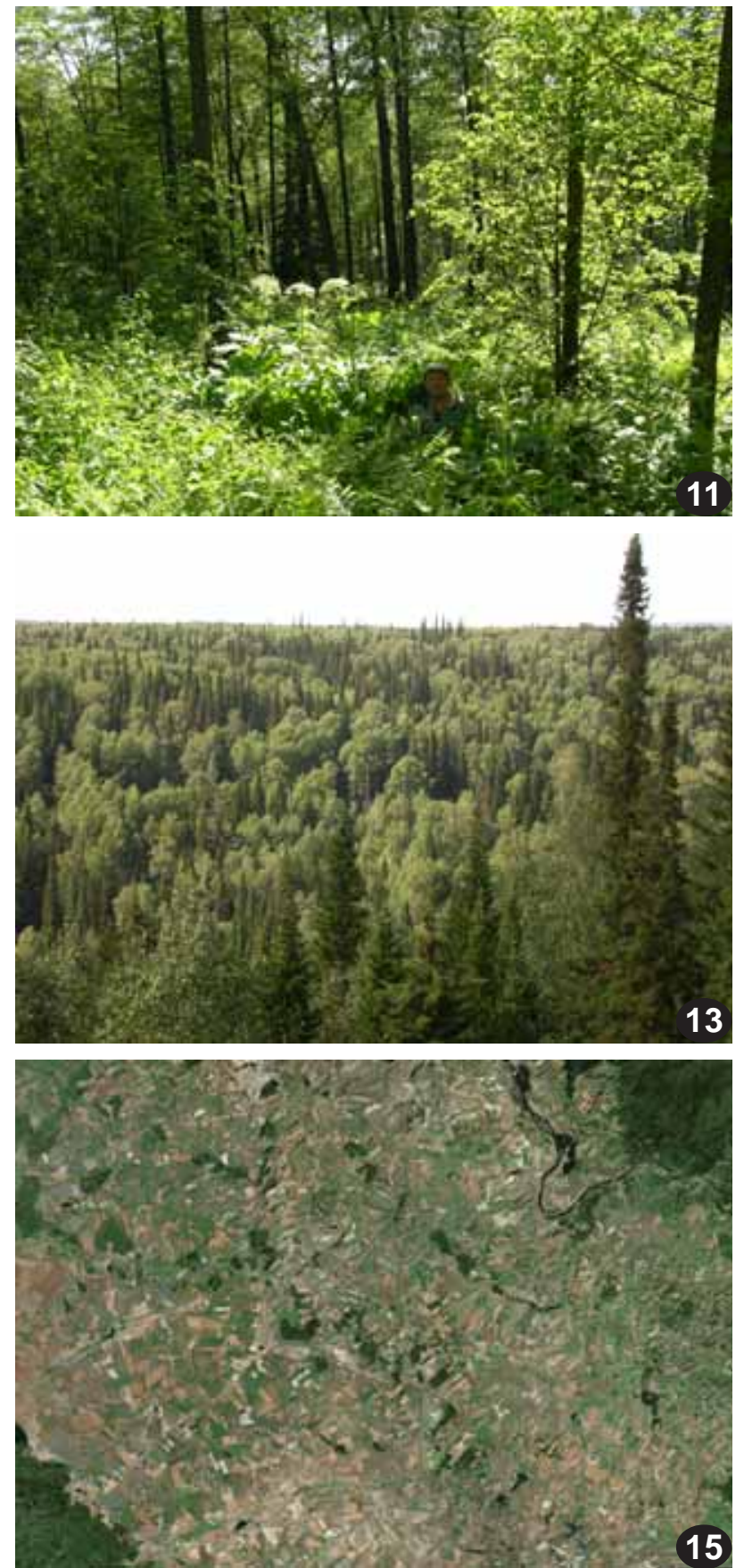

Figs. 11-12. Mountain Shoria, "Linden island": chernevaia taiga with abundant Tilia sibirica; spot with dense tree layer (Fig. 12) and a gap with tall-herbs (Fig. 11). Anomodon longifolius, Entodon schleicheri, Leucodon sciuroides grow here on Tilia trunks; Brachythecium rutabulum, Eurhynchium angustirete, Fissidens taxifolius on soil. Figs. 13-14. Salair Ridge, typical chernevaia taiga outside (Fig. 13) and inside (Fig. 14): alternation of tall-herb meadows with groups of Populus tremula and Abies sibirica. Figs. 15-17. Kuznetskaya depression. Space photo: agricultural lands and mines with only fragments of parent vegetation (Fig. 15, from http:// sasgis.ru). Forest-stepper landscape (Fig. 16). Petrophytic steppe on steep slopes (Fig. 17) provides habitats for Abietinella abietina, Rhytidium rugosum, Syntrichia ruralis, Tortella tortuosa, and Dicranum muehlenbeckii.
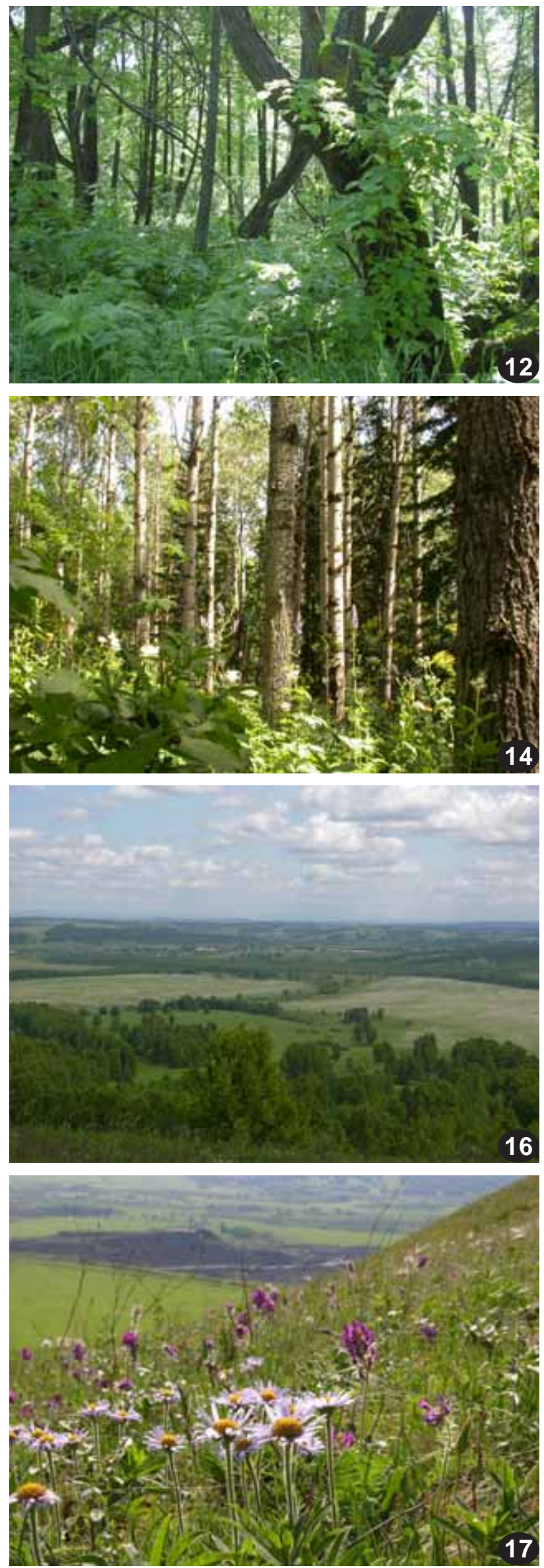

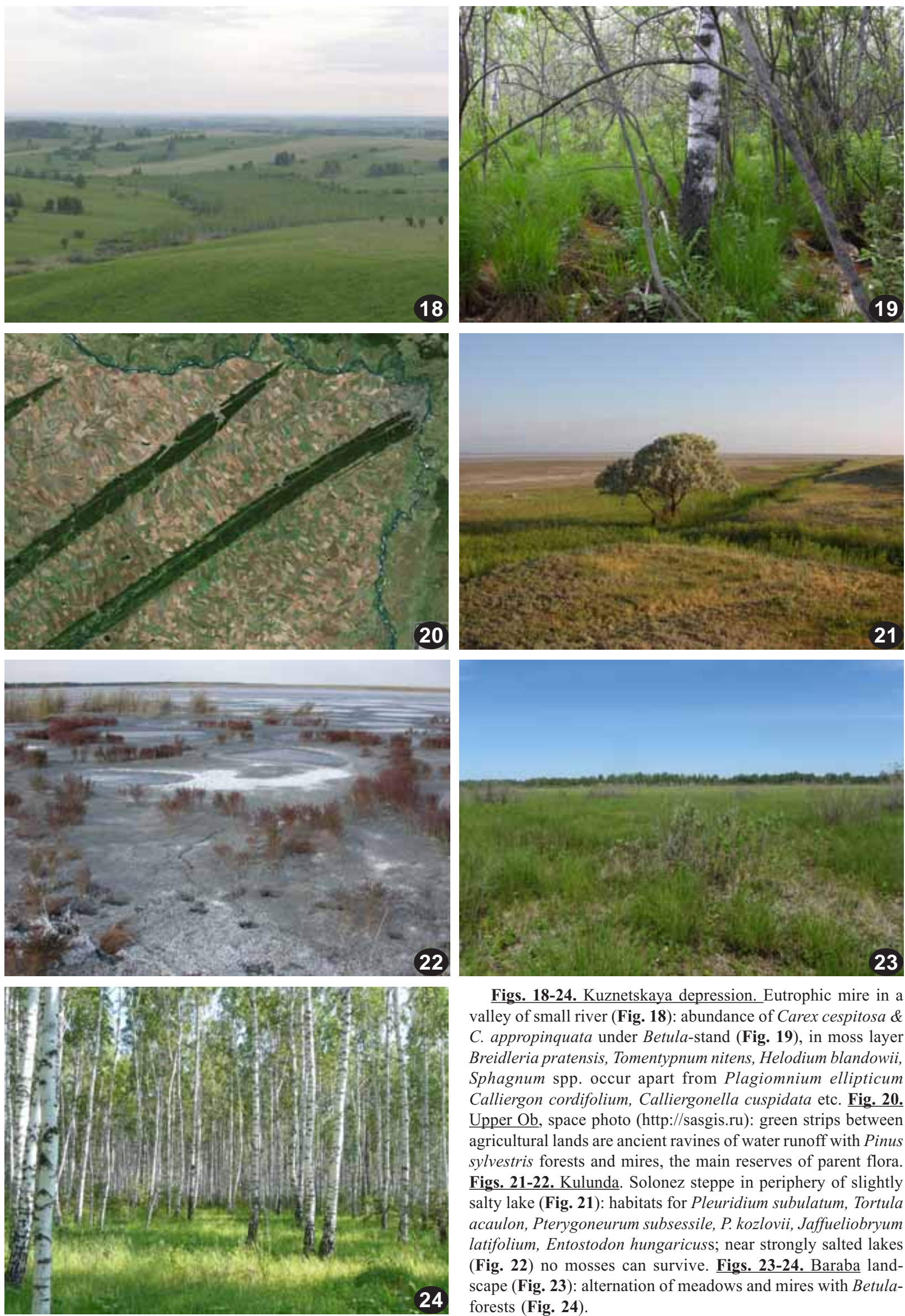

Figs. 18-24. Kuznetskaya depression. Eutrophic mire in a valley of small river (Fig. 18): abundance of Carex cespitosa \& C. appropinquata under Betula-stand (Fig. 19), in moss layer Breidleria pratensis, Tomentypnum nitens, Helodium blandowii, Sphagnum spp. occur apart from Plagiomnium ellipticum Calliergon cordifolium, Calliergonella cuspidata etc. Fig. 20. Upper Ob, space photo (http://sasgis.ru): green strips between agricultural lands are ancient ravines of water runoff with Pinus sylvestris forests and mires, the main reserves of parent flora. Figs. 21-22. Kulunda. Solonez steppe in periphery of slightly salty lake (Fig. 21): habitats for Pleuridium subulatum, Tortula acaulon, Pterygoneurum subsessile, P. kozlovii, Jaffueliobryum latifolium, Entostodon hungaricuss; near strongly salted lakes (Fig. 22) no mosses can survive. Figs. 23-24. Baraba landscape (Fig. 23): alternation of meadows and mires with Betulaforests (Fig. 24). 

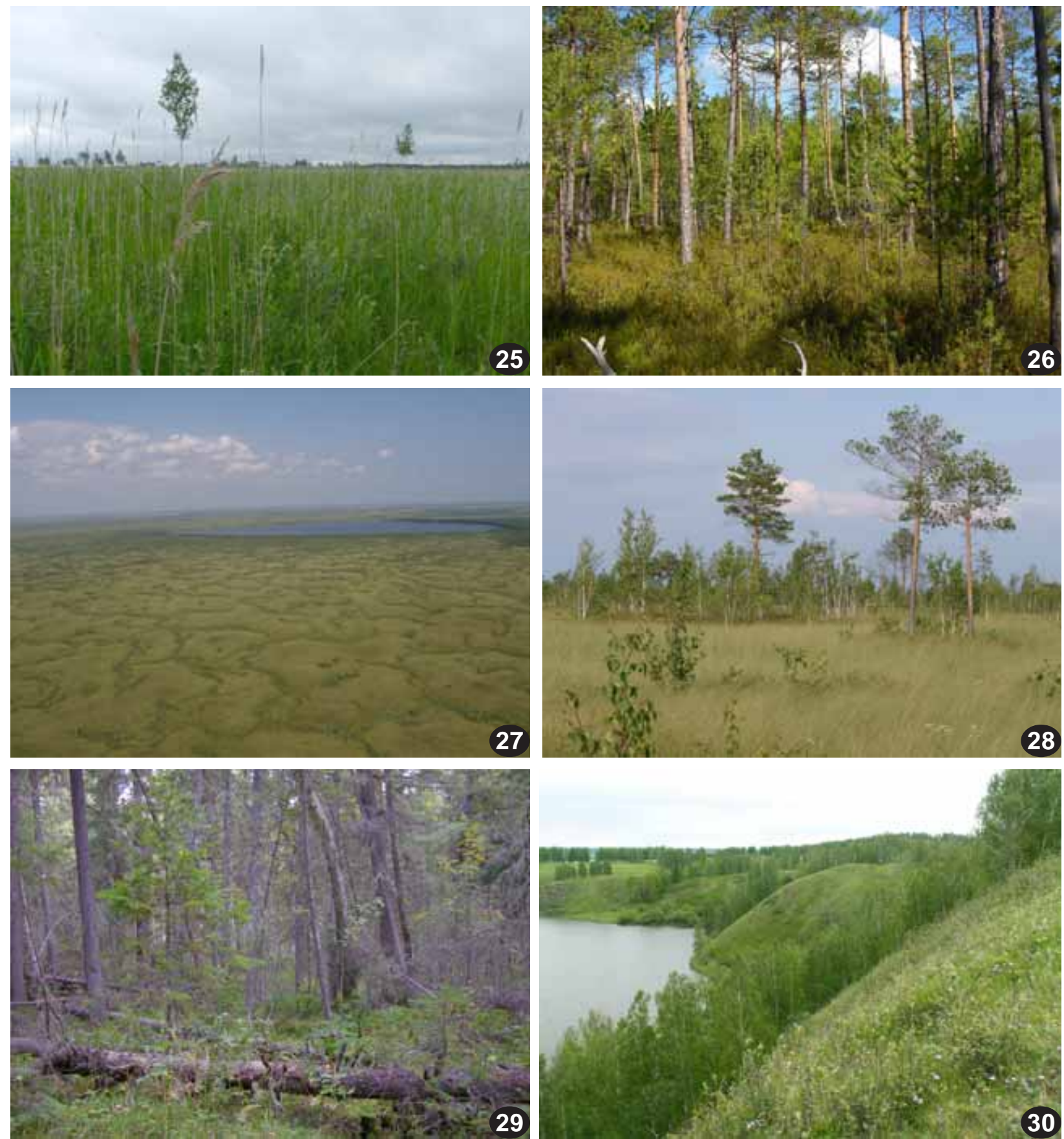

Figs. 25-26. SW West Siberian Plain. Eutrophic mire with Phragmites australis dominance (Fig. 25). Oligotrophic raised peat bogs (riams) (Fig. 26) are Pleistocene relicts in the landscape. Fig. 27-28. Great Vasyugan Swamp, view from helicopter (Fig. 27) and inside (Fig. 28): huge mire system from Carex-swamps and low Sphagnum-ridges with Pinus sylvestris and Betula. Fig. 29. South taiga in West Siberia is polydominant coniferous forest with well-developed and rich grass layer; moss cover is not closed, but rather diverse. By river valleys south taiga communities penetrate subtaiga zone to $54{ }^{\circ}$ N. Fig. 30-31. Transitional region, subtaiga zone. Rock outcrops and petrophytic steppe in river valley (Fig. 30). Native Betula-forest (Fig. 31): mosses are abundant on Betulatrunks (Pylaisia polyantha, Stereodon pallescens, Callicladium haldanianum, Platygyrium repens, Haplocladium microphyllum, etc.).

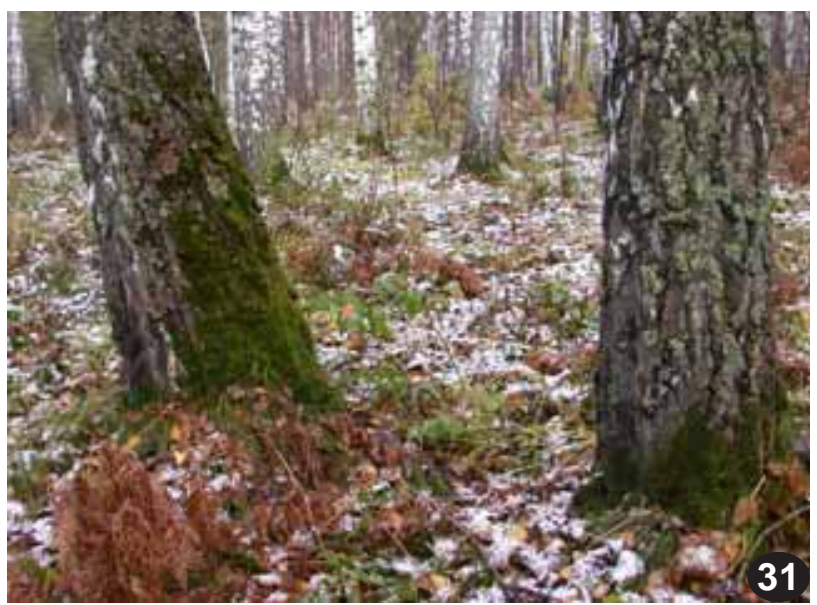


tion occupies non-sandy sites and is presented by a combination of small massifs of Betula-forests and different meadow and steppe communities (Lapshina, 1963; Makunina et al., 2010; Latchinsky \& Latchinskaya, 2012).

Kulunda is an accumulative plain within the Ob-Irtysh watershed. It is the lowest part of the study area with the mean altitudes of $100-140 \mathrm{~m}$. Its surface is formed by a thick layer (to $50-60 \mathrm{~m}$ ) of alluvial sediments. Shallow internal-drainage mineralized lakes are a characteristic feature of the region (Nikolaev, 1972, 1988; Nikolaev et al., 1982). The territory is located within the steppe zone (Ilina et al., 1985; Vandakurova, 1950).

Mosaic vegetation reflects differences in microrelief and soils. Zonal communities are steppe with Stipa spp. and Festuca valesiaca predominance. Solonez dry steppe are widespread. All watersheds of the territory are plowed; in some administrative districts arable lands take up to $80 \%$. Remaining steppe areas exist under strong pasturing; now they are mainly degraded and present poor communities of Artemisia austriaca, A. glauca, Festuca valesiaca. Halophyte communities are very diverse on this territory; they take place in depressions, in salt lake basins and river valleys (Korolyuk, 1999; Korolyuk et al., 2007). The most common species are Artemisia nitrosa, Puccinellia tenuissima, Limonium gmelinii, Halimione verrucifera, Salicornia perennans, Suaeda prostrate. In more wet places Hordeum brevisubulatum, Alopecurus arundinaceus, Agrostis stolonifera, Elytrigia repens, Juncus gerardii, Scorzonera parviflora, Plantago cornuti prevail. In the margins of overgrowing lake communities, Phragmites australis, Typha latifolia, Carex riparia, C. pseudocyperus, Thelypteris palustris, and Scolochloa festucacea are common. Forest communities are rather rare in the region. They occur on spots of winter snow accumulation - on concave downwind slopes of slightly elevated places and in small depressions (Korolyuk et al., 2008). The communities are formed by scrubby beech and aspen trees. Characteristic species of herb layer are Artemisia latifolia, Lathyrus pratensis, L. pisiformis, Achillea asiatica, Hieracium virosum, Achillea cartilaginea, Veronica longifolia, Kadenia dubia, Carex disticha, $C$. melanostachya). Sandy deposits of ancient ravines of water runoff ensure the existence of dry Pinus-forests with psammophyte species in the herb layer. The ancient ravines are covered with mire communities of Phragmites australis, swampy birch forests with Carex riparia, C. pseudocyperus, C. atherodes, Thelypteris palustris, Filipendula ulmaria.

Baraba is the alluvial drainless plain to the north of Kulunda. There is no distinct boundary between Baraba and Kulunda plains; usually they are delimited by the Bagan and Karasuk River watershed. The northern border of the forest-steppe zone is taken as the Baraba northern border; it matches with the northern limit of salted soils and is about $56^{\circ} \mathrm{N}$. Altitudes of the territory are 105-145 m. Baraba has well-expressed mesorelief formed by interspersed elevations and depressions about some hundred meters across, having 5-10 $\mathrm{m}$ in relative hight. The form of mesorelief elements varies from narrow and elongated in the western part to more or less roundish in the eastern part. There are a lot of shallow salted and fresh lakes on the territory.

Drained habitats are occupied by Betula-forests and grassland (meadow steppe in southern part) (Vagina, 1962; Kuminova et al., 1963; Korolyuk, 1993a,b; Korolyuk \& Kiprijanova, 2005). Mires take huge areas. Most common of them are eu- and mesotrophic sedge mires with abundance of Phragmites australis, Carex disticha, C. atherodes, C. omskiana, C. diandra, C. rostrata, $C$. riparia, Calamagrostis canescens. Oligotrophic raised peat bogs (riams) also occur, being considered as Pleistocene relicts. Bog massifs occur in small pan depressions in a forest-steppe landscape and have some hundred meters in diameter. They have a uniform composition and structure. Peat surface is covered by oligotrophic and mesotrophic Sphagnum species or by taiga mosses Pleurozium schreberi, Hylocomium splendens, Dicranum polysetum in more dry spots. A sparse tree layer is formed by undersized Pinus sylvestris of 6-10 m tall. In the herbshrub layer, Chamaedaphne calyculata, Ledum palustre, Vaccinium vitis-idaea, Carex globularis, Eriophorum vaginatum, Rubus chamaemorus are abundant (Valutskij, 2011). In the periphery of lake and mire basins, solonez and solonchak communities are common (Vagina, 1963; Korolyuk, 1999).

Vasyugan plain and adjacent areas. The central part of Ob-Irtysh watershed is a vast denudation-accumulative plain with mean altitudes $100-140 \mathrm{~m}$. Most part of the plain is the Great Vasyugan Swamp - the largest mire system in the world, $1500 \times 450 \mathrm{~km}$.

Swamp communities are diverse in the system. Eight main types by moisture and nutrient conditions result in corresponding plant communities (Liss \& Berezina, 1981; Lapshina et al., 2000; Pisarenko et al., 2011). Oligotrophic Sphagnum bogs are most widespread. In the southern part, mesotrophic sedge mires with Bryalean mosses cover take place (commonly called "Hypnum"-type bogs). To the south of the Great Vasyugan Swamp, the watershed habitats are covered by herbaceous Betula-forests; these forests are considered here to be primary (not secondary as Betula forest in many other areas). The zone formed by them is segregated in Siberia as the subtaiga zone (Gorodkov, 1915; Krylov, 1919; Khramov \& Valutsky, 1977; Ilina et al., 1985). Forests of Abies sibirica and Picea obovata grow here only in the river valleys.

To the north of the Great Vasyugan Swamp, the native vegetation of drained habitats is the south taiga: polydominant coniferous forest with herb cover with Carex macroura predominance (Latchinsky \& Korolyuk, 2012). Because of the mire landscape, the boundary between subtaiga and south taiga zones in the territory is conventional. 


\section{Species LisT}

Occurrence of species is shown by regions (for abbreviations see Table 1 and Fig. 2), according to the following scale: $\mathrm{w}=$ widespread $(>30$ localities $) ; \mathrm{c}=$ common (recorded from 16-30 localities); $\mathrm{sp}=$ sporadic $(6-15$ localities $) ; \mathrm{r}=$ rare (2-5 localities); $\mathrm{u}=$ unique (1 locality). For unique species the collecting point or reference [if it was published in Arctoa] is cited in the "Comments" section under the number given under the corresponding species name. Square brackets indicate species reported based on the literature data only, as no species were located, and comments are given also in these cases. The author specimens are in NSK. Column alt includes altitude interval within the studied territory. Three right columns specify species presence in administrative units: No - Novosibirsk Province, Ke Kemerovo Province, Al - Altaisky Territory outside Altai Mountains. At least one label from each region is in database "Herbarium specimens of Russian mosses" (http://arctoa.ru/Flora/basa.php).

Species

Abietinella Müll.Hal. abietina (Hedw.) M.Fleisch. (1)

Acaulon Müll.Hal. triquetrum (Spruce) Müll.Hal. (2)

\section{Aloina Kindb.}

brevirostris (Hook. \& Grev.) Kindb.(3) rigida (Hedw.) Limpr.

Amblystegium Bruch et al. radicale (P.Beauv.) Bruch et al. (4) serpens (Hedw.) Bruch et al.

Amphidium Schimp. lapponicum (Hedw.) Schimp. mougeotii (Bruch et al.) Schimp. (5)

Anacamptodon Brid. latidens (Besch.) Broth. (6)

Andreaea Hedw. rupestris Hedw.

Anomodon Hook. \& Taylor attenuatus (Hedw.) Huebener (7) longifolius (Brid.) Hartm. (8) rugelii (Müll.Hal. ) Keissl. viticulosus (Hedw.) Hook. \& Tayl. (9)

Atrichum P.Beauv. flavisetum Mitt. tenellum (Roehl.) Bruch et al. (10) undulatum (Hedw.) P.Beauv. (11)

Aulacomnium Schwägr. palustre (Hedw.) Schwägr. turgidum (Wahlenb.) Schwägr.

Barbula Hedw. convoluta Hedw. (12) unguiculata Hedw.

Bartramia Hedw. ithyphylla Brid. pomiformis Hedw. (13)

Brachytheciastrum Ignatov \& Huttunen velutinum (Hedw.) Ignatov \& Huttunen (14)

Brachythecium Bruch et al. (15) albicans (Hedw.) Bruch et al. (16) baicalense Ignatov (17) campestre (Müll.Hal. ) Bruch et al. (18) cirrosum (Schwägr.) Schimp. complanatum Broth. (19) erythrorrhizon Bruch et al. (20) mildeanum (Schimp.) Schimp. rivulare Bruch et al. rotaeanum De Not. rutabulum (Hedw.) Bruch et al. salebrosum (F.Weber \& D.Mohr) Bruch et al.

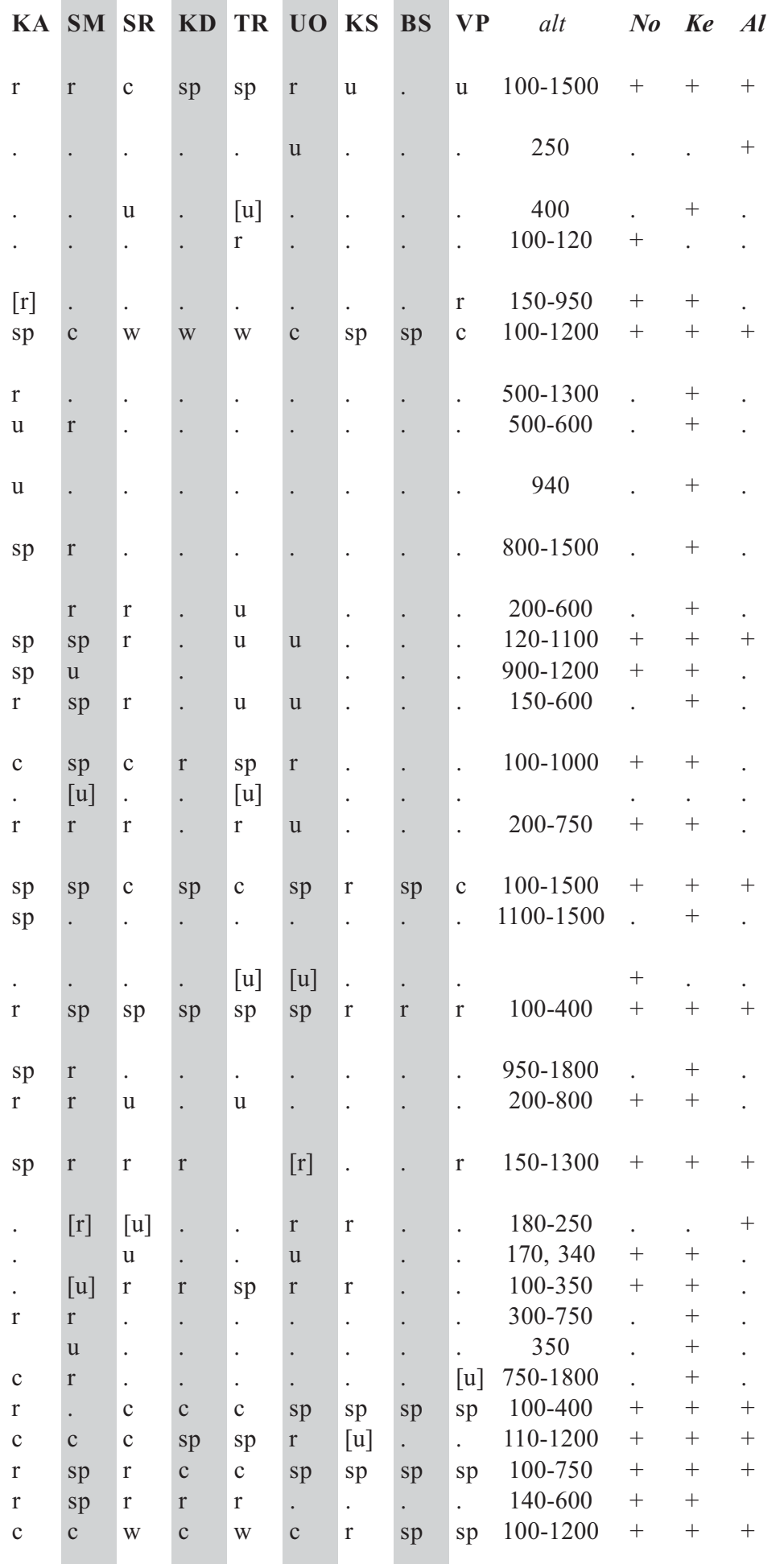


Species

Breidleria Loeske pratensis (W.D.J.Koch ex Spruce) Loeske (21) u

Bryhnia Kaurin brachycladula Cardot

Bryobrittonia R.S.Williams longipes (Mitt.) D.G.Horton (22)

Bryoerythrophyllum P.C.Chen recurvirostrum (Hedw.) P.C.Chen

Bryum Hedw. (23)

amblyodon Müll.Hal.

argenteum Hedw.

bimum (Schreb.) Turner

caespiticium Hedw.

capillare Hedw. (24)

creberrimum Taylor

cyclophyllum (Schwägr.) Bruch et al. (25)

elegans Nees (26)

funkii Schwaegr. (27)

intermedium (Brid.) Blandow (28)

lonchocaulon Müll.Hal.

moravicum Podp. (29)

neodamense Itzigs. (30)

pallens Sw. ex anon. (31)

pallescens Schleich. ex Schwägr. (32)

pseudotriquetrum (Hedw.) P.Gaertn., B.Mey. \& Scherb. sp

schleicheri DC.

subapiculatum Hampe. (33)

uliginosum (Brid.) Bruch et al.

weigelii Spreng. (34)

Bucklandiella Roiv.

microcarpa (Hedw.) Bednarek-Ochyra \& Ochyra sp sudetica (Funck) Bednarek-Ochyra \& Ochyra c

Buxbaumia Hedw. aphylla Hedw. (35)

Callicladium H.A.Crum haldanianum (Grev.) H.A.Crum

Calliergon (Sull.) Kindb cordifolium (Hedw.) Kindb. giganteum (Schimp.) Kindb. (36) megalophyllum Mikut. (37) richardsonii (Mitt.) Kindb.

Calliergonella Loeske cuspidata (Hedw.) Loeske lindbergii (Mitt.) Hedenäs

Campyliadelphus (Kindb.) R.S.Chopra chrysophyllus (Brid.) R.S.Chopra (38)

Campylidium (Kindb.) Ochyra calcareum (Crundw. \& Nyholm) Ochyra (39) sommerfeltii (Myrin) Ochyra

Campylium (Sull.) Mitt. protensum (Brid.) Kindb. stellatum (Hedw.) C.E.O.Jensen

Campylophyllum (Schimp.) M.Fleisch. halleri (Hedw.) M.Fleisch. (40)

Ceratodon Brid. purpureus (Hedw.) Brid.

Cinclidium Sw. stygium Sw. (41)

Cinclidotus P.Beauv. riparius (Host ex Brid.) Arn.

$\mathrm{sp}$
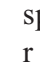

(n)
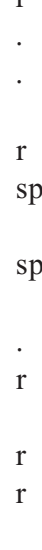

$\mathrm{u}$

sp
KA SM SR KD TR UO KS BS VP alt No Ke Al

120-300

$150-500$

400-600

$120-900$

$100-1200$

$100-1000$

sp $\quad 100-350$

100-400

250-1200

sp $100-1000$

150

250-1100

200-400

[r] 300-1100

200-500

250-800

150-200

$140-170$

[u]

$\mathrm{sp}$

$100-1300+++$

$360-800$

200-400

[u] $100-900$

600-1800

700-1800

$[\mathrm{u}]$

sp $\quad$ w

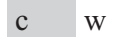

sp $\quad \mathrm{sp}$

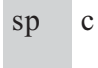

$100-800$

$r$

$\mathrm{sp}$

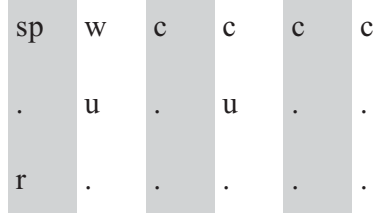


Species

Cirriphyllum Grout piliferum (Hedw.) Grout

Climacium F.Weber \& D.Mohr dendroides (Hedw.) F.Weber \& D.Mohr

Cnestrum I.Hagen schistii (F.Weber \& D.Mohr) I.Hagen

Conardia H.Rob. compacta (Drumm. ex Mull. Hal.) H. Rob. (42)

Cratoneuron (Sull.) Spruce filicinum (Hedw.) Spruce (43)

Cynodontium Bruch et al. polycarpon (Hedw.) Schimp. strumiferum (Hedw.) Lindb. tenellum (Schimp.) Limpr. (44)

Cyrtomnium Holmen hymenophylloides (Huebener) T.J.Kop.

Dichelyma Myrin falcatum (Hedw.) Myrin (45)

Dichodontium Schimp. pellucidum (Hedw.) Schimp. (46)

Dicranella (Müll.Hal.) Schimp. cerviculata (Hedw.) Schimp. crispa (Hedw.) Schimp. (47) heteromalla (Hedw.) Schimp. rufescens (Dicks.) Schimp. schreberiana (Hedw.) Hilf. ex H.A.Crum \& L.E.Anderson (48) subulata (Hedw.) Schimp. (49) varia (Hedw.) Schimp. (50)

Dicranodontium Bruch et al. denudatum (Brid.) E.Britton

Dicranum Hedw.

acutifolium (Lindb. \& Arnell) C.E.O.Jensen (51) r bonjeanii De Not.

brevifolium (Lindb.) Lindb. (52)

drummondii C.Muell. (53)

elongatum Schleich. ex Schwägr.

flagellare Hedw.

flexicaule Brid.

fragilifolium Lindb.

fuscescens Turner

laevidens R.S.Williams

leioneuron Kindb. (54)

majus Turner

montanum Hedw.

muehlenbeckii Bruch et al.

polysetum $\mathrm{Sw}$.

scoparium Hedw.

spadiceum J.E.Zetterst.

undulatum Schrad. ex Brid.

viride (Sull. et Lesq.) Lindb. (55)

Didymodon Hedw.

fallax (Hedw.) R.H.Zander

ferrugineus (Schimp. ex Besch.) M.O.Hill (56) icmadophilus (Schimp. ex Müll.Hal. ) R.H.Zander (57) . rigidulus Hedw.

tophaceus (Brid.) Lisa (58)

validus Limpr.

Discelium Brid. nudum (Dicks.) Brid.

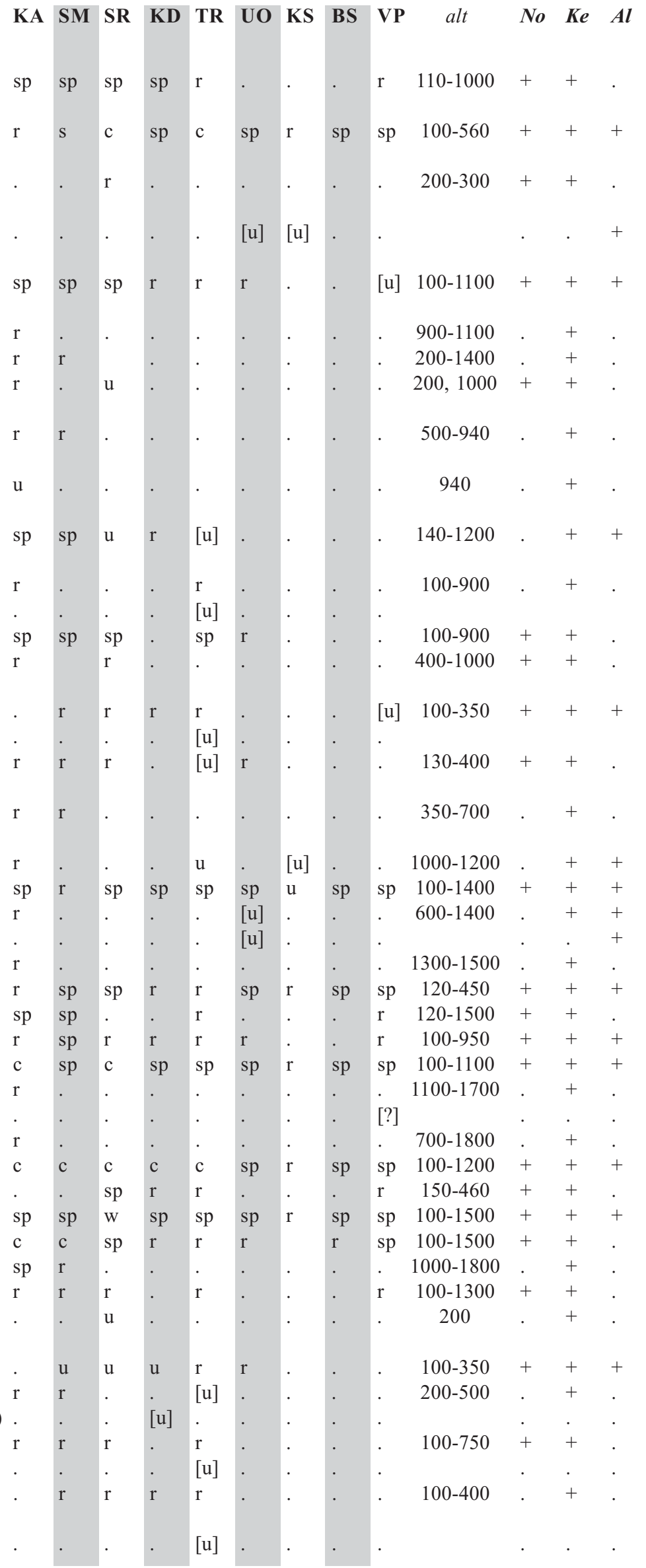


Species

Distichium Bruch et al. capillaceum (Hedw.) Bruch et al. inclinatum (Hedw.) Bruch et al. (59)

Ditrichum Timm ex Hampe cylindricum (Hedw.) Grout (60) flexicaule (Schwägr.) Hampe heteromallum (Hedw.) E.Britton (61) pusillum (Hedw.) Hampe (62)

Drepanium C.E.O.Jensen recurvatum (Lindb. \& Arnell) G.Roth

Drepanocladus (Müll.Hal.) G.Roth aduncus (Hedw.) Warnst.

polygamus (Bruch et al.) Hedenäs sendtneri (Schimp. ex H.Muell.) Warnst. (63)

Encalypta Hedw. (64)

ciliata Hedw.

pilifera Funck

procera Bruch

rhaptocarpa Schwägr. (65)

trachymitria Ripart (66)

vulgaris Hedw. (67)

Entodon Müll.Hal.

concinnus (De Not.) Paris (68)

schleicheri (Schimp.) Demet. (69)

Entosthodon Schwägr.

hungaricus (Boros) Loeske

Eurhynchiastrum Ignatov \& Huttunen pulchellum (Hedw.) Ignatov \& Huttunen

Eurhynchium Bruch et al. angustirete (Broth.) T.J.Kop.

Fabronia Raddi ciliaris (Brid.) Brid. (70)

Fissidens Hedw. adianthoides Hedw. (71) bryoides Hedw.

exilis Hedw. (72)

osmundoides Hedw. (73)

taxifolius Hedw. (74)

viridulus (Sw.) Wahlenb. (75)

Fontinalis Hedw.

antipyretica Hedw.

hypnoides Hartm. (76)

Funaria Hedw.

hygrometrica Hedw.

Grimmia Hedw.

alpestris (F.Weber \& D.Mohr) Schleich. anodon Bruch et al. (77)

anomala Hampe ex Schimp.

caespiticia (Brid.) Jur.

elatior Bruch ex Bals.-Criv. \& De Not.

funalis (Schwägr.) Bruch et al. (78)

incurva Schwägr.

laevigata (Brid.) Brid.

longirostris Hook.

muehlenbeckii Schimp.

pulvinata (Hedw.) Sm. (79)

reflexidens Müll.Hal.

teretinervis Limpr.

tergestina Tomm. ex Bruch et al. (80)

unicolor Hook. (81)

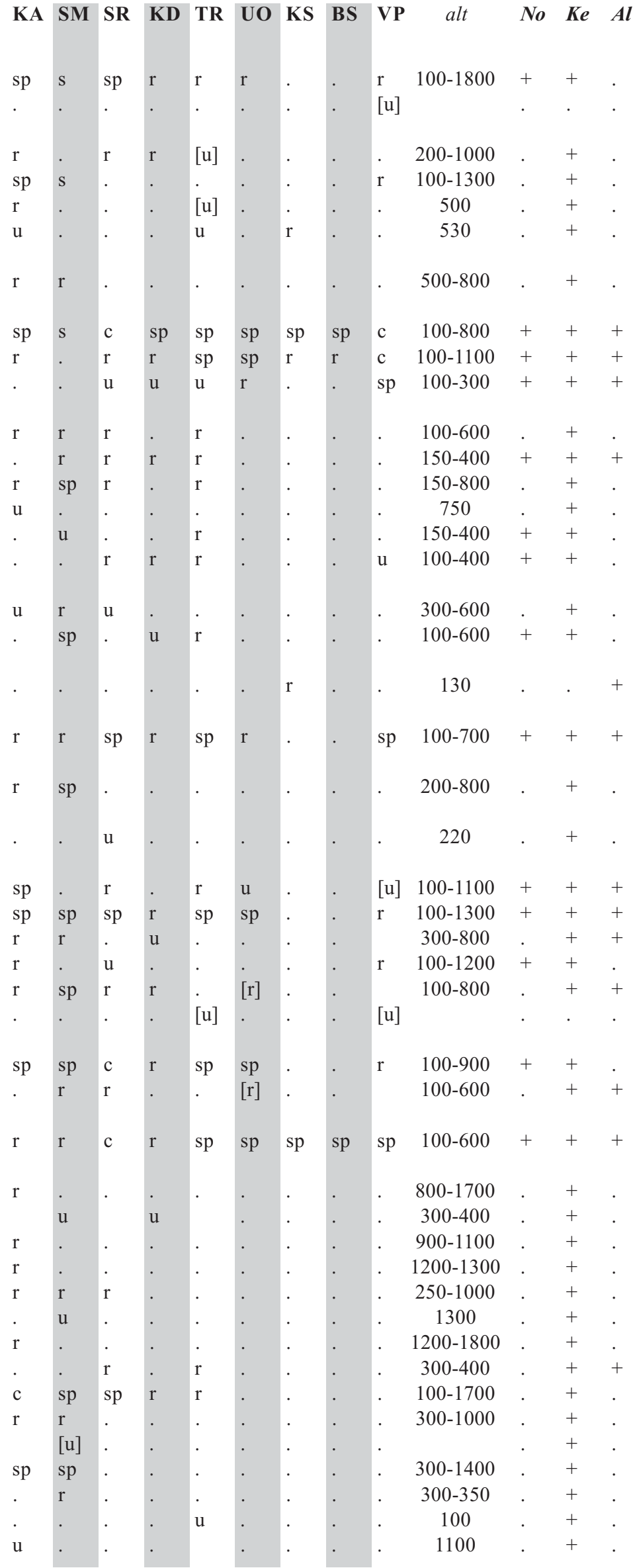


Species

Gymnostomum Nees \& Hornsch. aeruginosum $\mathrm{Sm}$. (82)

Hamatocaulis Hedenäs lapponicus (Norrl.) Hedenäs vernicosus (Mitt.) Hedenäs (83)

Haplocladium (Müll.Hal.) Müll.Hal. angustifolium (Hampe \& Müll.Hal. ) Broth. (84) u microphyllum (Hedw.) Broth.

Hedwigia P.Beauv. ciliata (Hedw.) P.Beauv.

Helodium Warnst. blandowii (F.Weber \& D.Mohr) Warnst. (85)

Herzogiella Broth. striatella (Brid.) Z.Iwats. (86) turfacea (Lindb.) Z.Iwats. (87)

Heterocladium Bruch et al. dimorphum (Brid.) Bruch et al. (88)

Homalia Brid. trichomanoides (Hedw.) Bruch et al. (89)

Homomallium (Schimp.) Loeske incurvatum (Schrad. ex Brid.) Loeske

Hygroamblystegium Loeske humile (P.Beauv.) Vanderp., Goffinet \& Hedenäs (90) [u] tenax (Hedw.) Jenn. (91) varium (Hedw.) Moenk.

Hygrohypnella Ignatov \& Ignatova ochracea (Turner ex Wilson) Ignatov \& Ignatova $\mathrm{sp}$

Hygrohypnum Lindb. luridum (Hedw.) Jenn.

Hylocomiastrum Broth. pyrenaicum (Spruce) M.Fleisch. umbratum (Hedw.) M.Fleisch.

Hylocomium Bruch et al. splendens (Hedw.) Bruch et al.

Hymenoloma Ochyra crispulum (Hedw.) Ochyra

Hypnum Hedw. cupressiforme Hedw. (92)

Isopterygiopsis Z.Iwats. alpicola (Lindb. \& Arnell) Hedenaes (93) muelleriana (Schimp.) Z.Iwats. (94) pulchella (Hedw.) Iwats. (95)

Iwatsukiella W.R.Buck \& H.A.Crum leucotricha (Mitt.) R.W.Buck \& H.A.Crum (96) r Jaffueliobryum Thér. latifolium (Lindb. \& Arnell) Ther. (97)

Kiaeria I.Hagen blyttii (Bruch et al.) Broth. falcata (Hedw.) I.Hagen starkei (F.Weber \& D.Mohr) I.Hagen

Leptobryum (Bruch et al.) Wilson pyriforme (Hedw.) Wils.

Leptodictyum (Schimp.) Warnst. riparium (Hedw.) Warnst.

Lescuraea Bruch et al. incurvata (Hedw.) E.Lawton (98) patens Lindb. saxicola (Bruch et al.) Molendo secunda Arnell

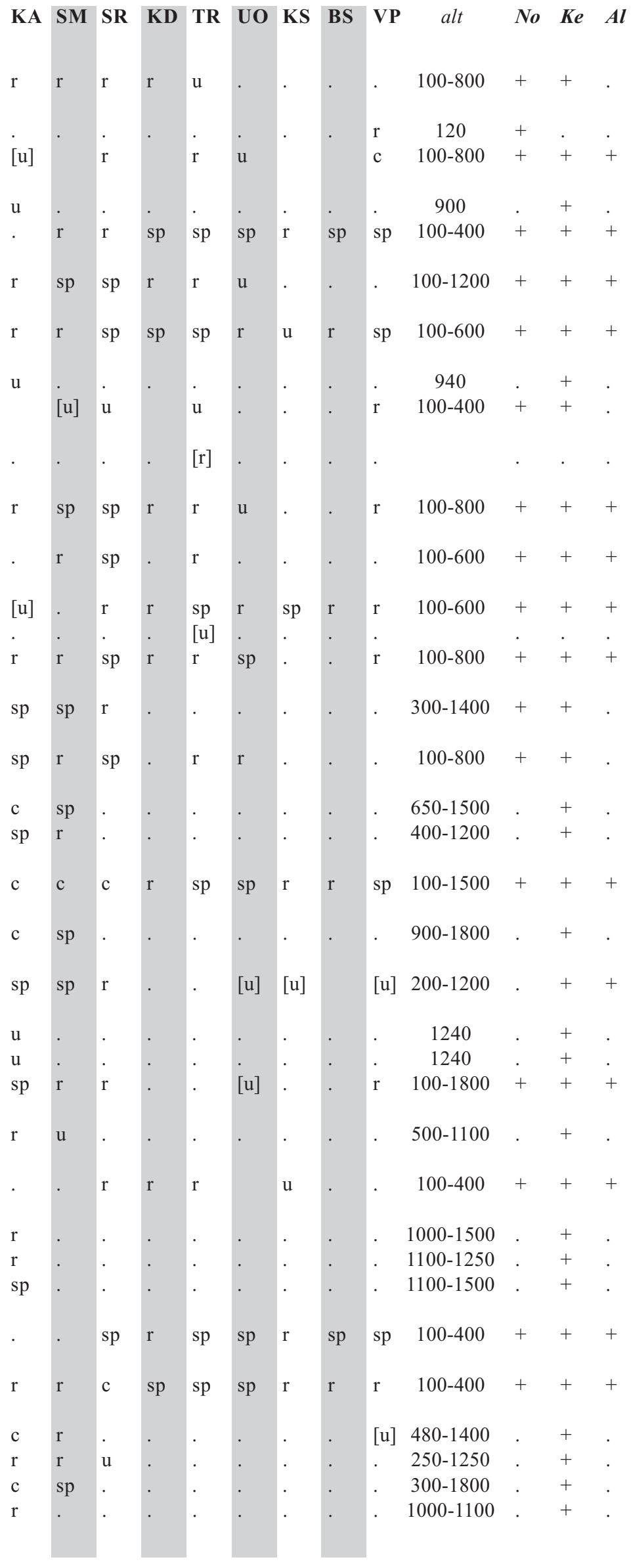


Species

Leskea Hedw. polycarpa Hedw.

Leucodon Schwägr. sciuroides (Hedw.) Schwägr. (99)

Meesia Hedw.

longiseta Hedw.

triquetra (Jolycl.) Angstr. (100)

uliginosa Hedw. (101)

Microbryum Schimp. curvicollum (Hedw.) R.H.Zander (102)

Mnium Hedw.

lycopodioides Schwägr. marginatum (Dicks.) P.Beauv. spinosum (Voit) Schwägr. spinulosum Bruch et al. (103) stellare Hedw. thomsonii Schimp.

Myrinia Schimp. pulvinata (Wahlenb.) Schimp.

Myurella Bruch et al.

julacea (Schwägr.) Bruch et al. (104)

sibirica (Müll.Hal. ) Reimers

tenerrima (Brid.) Lindb. (105)

Myuroclada Besch. maximowiczii (G.G.Borshch.) Steere \& W.B.Schofield

Neckera Hedw. pennata Hedw.

Niphotrichum (Bednarek-Ochyra) Bednarek-Ochyra \& Ochyra canescens (Hedw.) Bednarek-Ochyra \& Ochyra (106) c

Ochyraea Váňa duriuscula (De Not.) Ignatov \& Ignatova

Oligotrichum DC. hercynicum (Hedw.) Lam. \& DC.

Oncophorus (Brid.) Brid. virens (Hedw.) Brid. (107) wahlenbergii Brid.

Orthothecium Bruch et al. intricatum (Hartm.) Bruch et al. (108)

Orthotrichum Hedw. alpestre Hornsch. ex Bruch et al. (109) anomalum Hedw. cupulatum Brid. obtusifolium Brid. pallens Bruch ex Brid. (110) pellucidum Lindb. (111) rupestre Schleich. ex Schwägr. (112) speciosum Nees

Oxyrrhynchium (Schimp.) Warnst. hians (Hedw.) Loeske (113)

Oxystegus (Lindb. ex Limpr.) Hilp. tenuirostris (Hook. \& Tayl.) A.J.E.Sm.

Paludella Brid. squarrosa (Hedw.) Brid. (114)

Palustriella Ochyra commutata (Hedw.) Ochyra (115) decipiens (De Not.) Ochyra falcata (Brid.) Hedenäs (116)

Paraleucobryum (Limpr.) Loeske enerve (Thed.) Loeske (117) longifolium (Hedw.) Loeske (118)
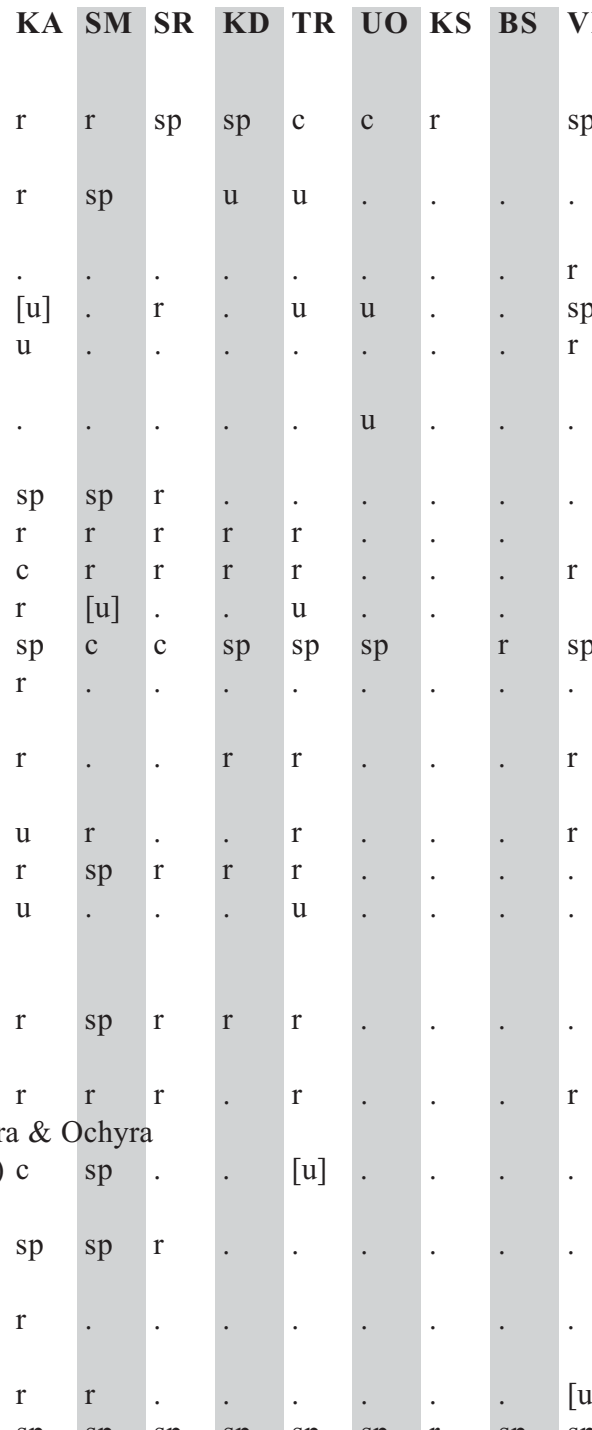

$\mathrm{sp}$

$100-600$

150

$150-900$

r $\quad 150-1100$

250

250-150

100-600

150-1200

150-1800

sp $100-1300$

500-800

100-600

120-750

100-800

200, 750

$100-60$

100-600

100-1550

300-1100

1100-1700

[u] 600-1300

100-1300
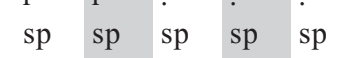

750

270

100-1100

200-750

sp

$\mathrm{sp}$

$100-95$$$
200
$$

300

100-900

1501100

200-1400

150-900

150-1000

560-1100

200

1600

200-1800

No Ke Al 
Species

Philonotis Brid. (119)

caespitosa Jur.

fontana (Hedw.) Brid. (120)

seriata Mitt.

Physcomitrella Bruch et al.

patens (Hedw.) Bruch et al. (121)

Physcomitrium (Brid.) Brid.

eurystomum Sendtn. (122)

pyriforme (Hedw.) Hampe (123)

Plagiomnium T.J.Kop. affine (Bland.) T.J.Kop. (124)

confertidens (Lindb. \& Arnell) T.J.Kop. (125)

cuspidatum (Hedw.) T.J.Kop.

drummondii (Bruch \& Schimp.) T.J.Kop. (126)

ellipticum (Brid.) T.J.Kop.

medium (Bruch et al.) T.J.Kop.

rostratum (Schrad.) T.J.Kop. (127)

Plagiopus Brid.

oederianus (Sw.) H.A.Crum \& L.E.Anderson (128) r

Plagiothecium Bruch et al.

cavifolium (Brid.) Z.Iwats. (129)

denticulatum (Hedw.) Bruch et al.

laetum Bruch et al. (130)

latebricola Bruch et al. (131)

Platydictya Berk.

jungermannioides (Brid.) H.A.Crum (132)

Platygyrium Bruch et al.

repens (Brid.) Bruch et al.

Pleuridium Rabenh.

subulatum (Hedw.) Rabenh. (133)

Pleurozium Mitt.

schreberi (Brid.) Mitt.

Podperaea Z.Iwats. \& Glime krylovii (Podp.) Z.Iwats. \& Glime (134)

Pogonatum P.Beauv.

dentatum (Brid.) Brid.

urnigerum (Hedw.) P.Beauv. (135)

Pohlia Hedw.

annotina (Hedw.) Lindb. (136)

cruda (Hedw.) Lindb.

drummondii (Müll.Hal. ) A.L.Andrews

elongata Hedw. (137)

longicollis (Hedw.) Lindb. (138)

melanodon (Brid.) A.J.Shaw (139)

nutans (Hedw.) Lindb.

obtusifolia (Vill. ex Brid.) L.F.Koch (140)

proligera (Kindb.) Lindb. ex Broth.

sphagnicola (Bruch et al.) Broth. (141)

wahlenbergii (F.Weber \& D.Mohr) A.L.Andrews

Polytrichastrum G.L.Sm.

alpinum (Hedw.) G.L.Sm. (142)

formosum (Hedw.) G.L.Sm. (143)

longisetum (Sw. ex Brid.) G.L.Sm. (144)

pallidisetum (Funck) G.L.Sm. (145)

sexangulare (Florke ex Brid.) G.L.Sm.

Polytrichum Hedw.

commune Hedw. (146)

juniperinum Hedw.

piliferum Hedw.

strictum Brid. (147)

;

u

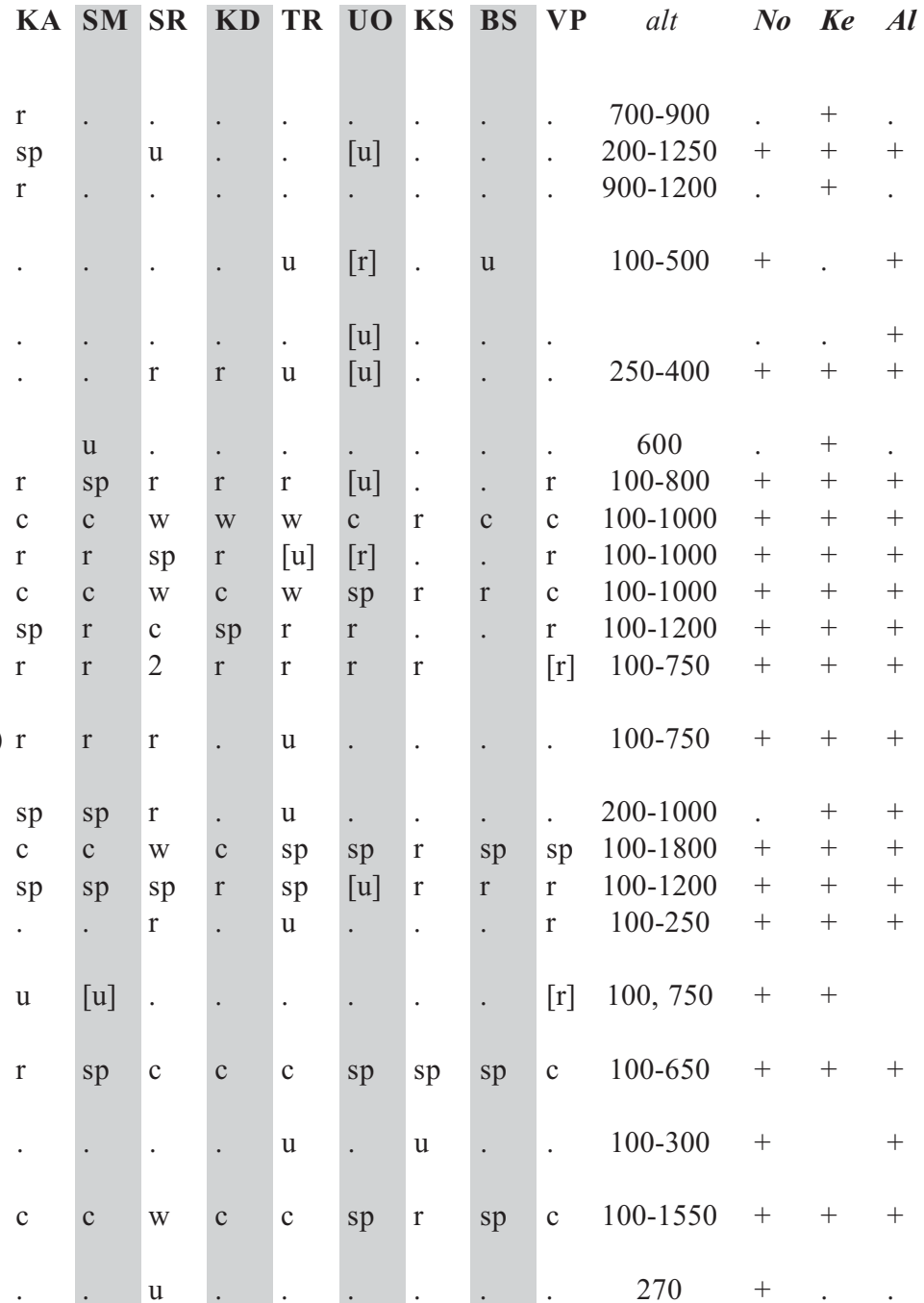

[u]

$\begin{array}{llll}{[\mathrm{u}]} & \cdot & \cdot & \cdot \\ \mathrm{sp} & \mathrm{sp} & \mathrm{r} & \mathrm{sp}\end{array}$

$\mathrm{sp}$

$\mathrm{r}$

[u]

[u] sp $\quad$ sp $\quad$ sp $\quad$ sp

$\mathrm{sp}$

sp

sp

$\mathrm{c} \quad \mathrm{sp}$

u

$\mathrm{sp}$

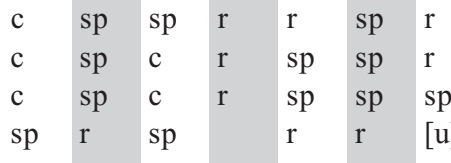

[u]

$\mathrm{sp} \quad \mathrm{sp}$

$\mathrm{sp}$

[u] $\mathrm{cp}$
500-1800

100-1800

700-900

200-1250

$100-50$

$250-40$

$100-1000+++$

$100-1000+++$

$100-1000+++$

$100-1200+++$

100-750 + + +

200-1000

.

$100-1700+++$

900-1700

200

550-600

100-200

100-1500

1100

100-350

[r]

[100-900] ++

$100-1300++\quad+$ 400-1200 . + + $120-1500+++$

550

1100-1700. +
250-1800. ++ 
Species

Pseudephemerum (Lindb.) I.Hagen nitidum (Hedw.) Loeske (148)

Pseudobryum (Kindb.) T.J.Kop. cinclidioides (Huebener) T.J.Kop.

Pseudocalliergon (Limpr.) Loeske lycopodioides (Brid.) Hedenäs trifarium (F.Weber \& D.Mohr) Loeske (149)

Pseudoleskeella Kindb.

catenulata (Brid. ex Schrad.) Kindb.

nervosa (Brid.) Nyholm (150)

papillosa (Lindb.) Kindb. (151)

rupestris (Berggr.) Hedenäs \& L.Söderstr. (152) tectorum (Funck ex Brid.) Kindb. ex Broth.

Pterigynandrum Hedw. filiforme Hedw. (153)

Pterygoneurum Jur. kozlovii Laz.

ovatum (Hedw.) Dixon subsessile (Brid.) Jur. (154)

Ptilium De Not. crista-castrensis (Hedw.) De Not.

Pylaisia Bruch et al. polyantha (Hedw.) Bruch et al. selwynii Kindb.

Rhabdoweisia Bruch et al. crispata (Dicks. ex With.) Lindb.

Rhizomnium (Broth.) T.J.Kop. andrewsianum (Steere) T.J.Kop. (155) magnifolium (Horik.) T.J.Kop. pseudopunctatum (Bruch \& Schimp.) T.J.Kop. punctatum (Hedw.) T.J.Kop. (156)

Rhodobryum (Schimp.) Limpr. ontariense (Kindb.) Kindb. (157) roseum (Hedw.) Limpr.

Rhynchostegium Bruch et al. arcticum (I.Hagen) Ignatov \& Huttunen rotundifolium (Scop. ex Brid.) Bruch et al. (158)

Rhytidiadelphus (Limpr.) Warnst. subpinnatus (Lindb.) T.J.Kop. (159) triquetrus (Hedw.) Warnst.

Rhytidium (Sull.) Kindb. rugosum (Hedw.) Kindb. (160)

Saelania Lindb. glaucescens (Hedw.) Broth.

Sanionia Loeske uncinata (Hedw.) Loeske

Schistidium Bruch et al. (161) apocarpum (Hedw.) Bruch et al. (162) boreale Poelt crenatum H.H.Blom dupretii (Ther.) W.A.Weber lancifolium (Kindb.) H.H.Blom obscurum H.H.Blom, Koeckinger \& Ignatova (163) . papillosum Culm. platyphyllum (Mitt.) Perss. (164) pulchrum H.H.Blom rivulare (Brid.) Podp. (165) sibiricum Ignatova \& H.H.Blom (166) sinensiapocarpum (Müll.Hal. ) Ochyra (167) submuticum Broth. ex H.H.Blom (168) tenuinerve Ignatova \& H.H.Blom (169)

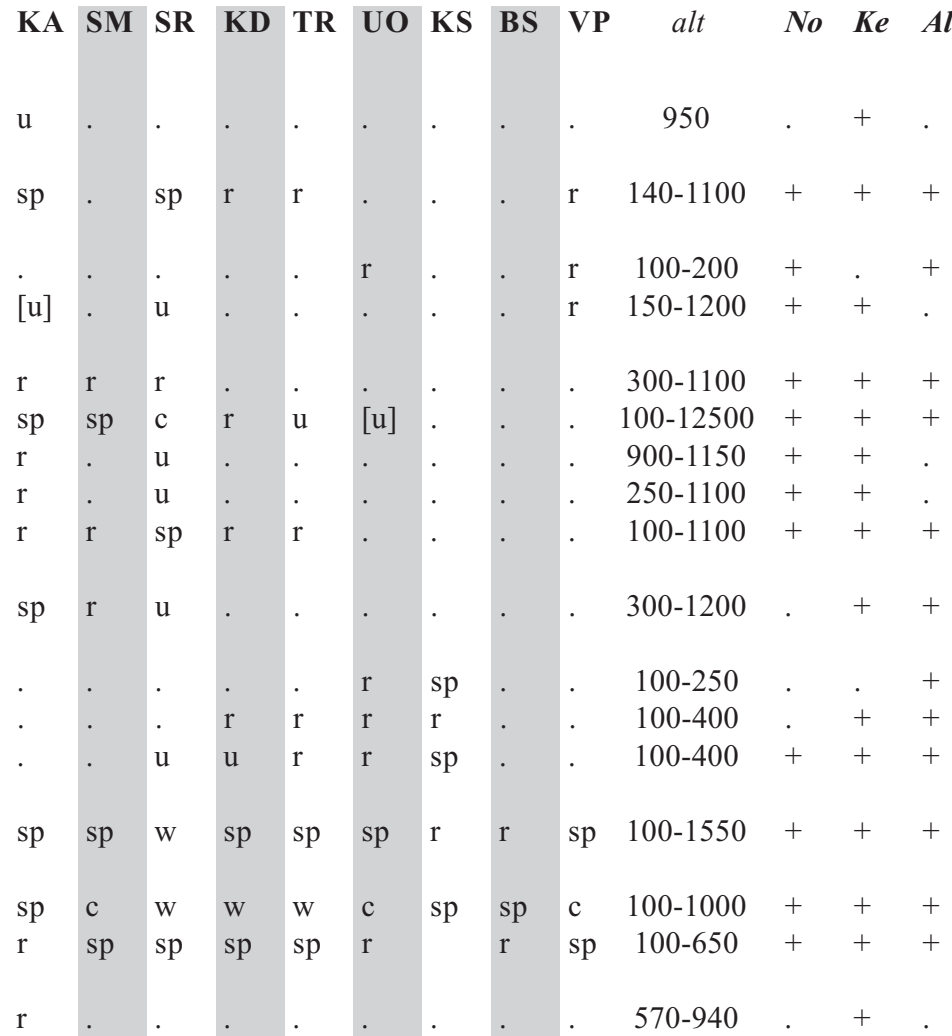

10

400-1200

100-1400

100-1200

200-600

$100-1100$

$360-1300$

500-750

140-1200

$\mathrm{sp}$

p

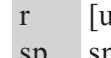

$u$

$\begin{array}{llllll}\mathrm{sp} & \mathrm{sp} & \mathrm{c} & \mathrm{sp} & \mathrm{r} & {[\mathrm{u}]}\end{array}$

150-1500

100-80

w
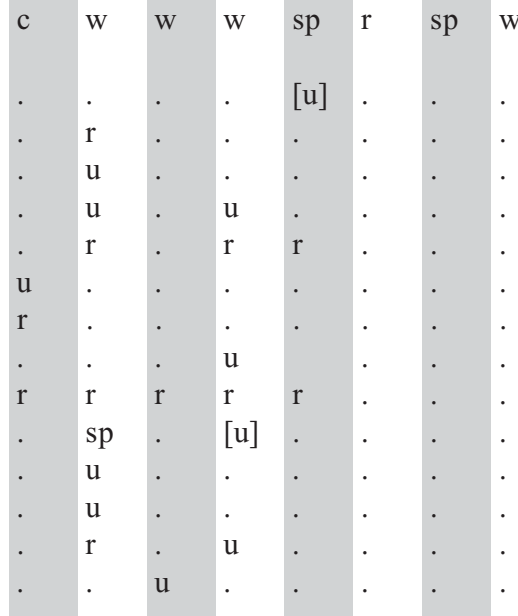
Species

Schistostega D.Mohr

pennata (Hedw.) F.Weber \& D.Mohr (170)

Sciuro-hypnum (Hampe) Hampe

altaicum (Ignatov) Ignatov

curtum (Lindb.) Ignatov

ornellanum (Molendo) Ignatov \& Huttunen

plumosum (Hedw.) Ignatov \& Huttunen (171)

populeum (Hedw.) Ignatov \& Huttunen reflexum (Starke) Ignatov \& Huttunen

starkei (Brid.) Ignatov \& Huttunen

Scorpidium (Schimp.) Limpr.

cossonii (Schimp.) Hedenäs (172)

revolvens (Sw. ex anon.) Rubers (173)

scorpioides (Hedw.) Limpr.

Seligeria Bruch et al.

brevifolia (Lindb.) Lindb. (174)

campylopoda Kindb. (175)

donniana (Sm.) Müll.Hal. (176)

pusilla (Hedw.) Bruch et al. (177)

tristichoides Kindb. (178)

Serpoleskea (Limpr.) Loeske confervoides (Brid.) Loeske subtilis (Hedw.) Loeske (179)

\section{Sphagnum L.}

angustifolium (C.E.O.Jensen ex Russow) C.E.O.Jensen sp aongstroemii Hartm. (180)

balticum (Russow) C.E.O.Jensen (181)

capillifolium (Ehrh.) Hedw.

centrale C.E.O.Jensen (182)

compactum Lam. \& DC. (183)

contortum Schultz (184)

cuspidatum Ehrh. ex Hoffm. (185)

fallax (H.Klinggr.) H.Klinggr. (186)

fimbriatum Wilson (187)

flexuosum Dozy \& Molk. (188)

fuscum (Schimp.) H.Klinggr.

girgensohnii Russow (189)

inundatum Russow (190)

jensenii H. Lindb. (191)

lindbergii Schimp. ex Lindb.

magellanicum Brid.

majus (Russow) C.E.O.Jensen (192)

obtusum Warnst. (193)

palustre L. (194)

papillosum Lindb.

platyphyllum (Lindb. ex Braithw.) Warnst. (195)

pulchrum (Lindb. ex Braithw.) Warnst. (196)

riparium Angstr. (197)

rubellum Wilson (198)

russowii Warnst.

squarrosum Crome (199)

subfulvum Sjoers (200)

subnitens Russow \& Warnst. (201)

subsecundum Nees

tenellum (Brid.) Pers. ex Brid. (202)

teres (Schimp.) Angstr. (203)

warnstorfii Russow

wulfianum Girg. (204)

Splachnum Hedw.

ampullaceum Hedw. (205)

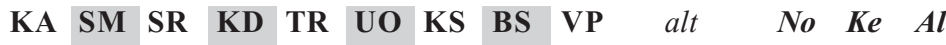

$300-1000++$
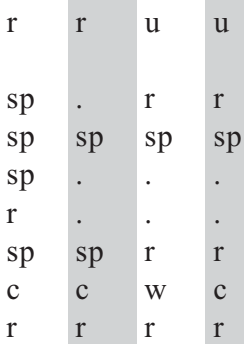

$[\mathrm{r}]$

200,900

900

sp

200-700 + + +

$100-1000+++$

$350-1100 \cdot+$.

1050-1200 . + +

200-1200+ +

100-1250 + + +

$100-1300+++$

750

750

750

300-1200 + + +

100-650 + + +

$100-1200++$

[900]

100-900

$100-1400$

$100-1200$

150-900

$150-900$

130

100-900

100-250

$150-1400$

$100-1400$

100-1200

[u] [u]

100-1200

150

100-900

150,900

$150-900$

$150-900$

$150-900$

150

150,800

[u]

150-250

[u] sp 100-1400

r sp 100-1400

sp $150-750$

[u] 130

900

sp $\quad 150-1200$

$$
900
$$

100-1100

$100-1200$

150-250

150 
Species

Stereodon (Brid.) Mitt. pallescens (Hedw.) Mitt. plicatulus Lindb.

vaucheri (Lesq.) Lindb. ex Broth.

Straminergon Hedenäs stramineum (Dicks. ex Brid.) Hedenäs (206)

Syntrichia Brid.

norvegica $\mathrm{F}$.Weber ruralis (Hedw.) F.Weber \& D.Mohr (207)

Taxiphyllum M.Fleisch. wissgrillii (Garov.) Wijk \& Margad.

Tayloria Hook. lingulata (Dicks.) Lindb. (208)

Tetraphis Hedw. pellucida Hedw. (209)

Tetraplodon Bruch et al. mnioides (Hedw.) Bruch et al.

Thamnobryum Nieuwl. neckeroides (Hook.) E.Lawton (210)

Thuidium Bruch et al. assimile (Mitt.) A.Jaeger recognitum (Hedw.) Lindb.

Timmia Hedw.

bavarica Hessl. (211)

comata Lindb. et H.Arnell (212) megapolitana Hedw.

Tomentypnum Loeske nitens (Hedw.) Loeske (213)

Tortella (Müll.Hal.) Limpr. alpicola Dixon (214) fragilis (Hook. \& Wils.) Limpr. (215) inclinata (R.Hedw.) Limpr. (216) tortuosa (Hedw.) Limpr.

Tortula Hedw.

acaulon (With.) R.H.Zander (217)

hoppeana (Schultz) Ochyra

modica R.H.Zander

mucronifolia Schwägr.

muralis Hedw. (218)

obtusifolia (Schwägr.) Mathieu (219)

truncata (Hedw.) Mitt.

Trachycystis T.J.Kop. ussuriensis (Maack \& Regel) T.J.Kop. (220)

Trichostomum Bruch crispulum Bruch (221)

Ulota D.Mohr rehmannii Jur. (222)

Warnstorfia Loeske exannulata (Bruch et al.) Loeske (223) fluitans (Hedw.) Loeske (224) pseudostraminea (Müll.Hal. ) Tuom. \& T.J.Kop

Weissia Hedw. brachycarpa (Nees \& Hornsch.) Jur. (225) controversa Hedw. (226) longifolia Mitt. (227)

Zygodon Hook. \& Taylor sibiricus Ignatov, Ignatova, Z.Iwats. \& B.C.Tan (228)

(1)
KA SM SR KD TR UO KS BS VP alt No Ke Al

$\mathrm{sp}$

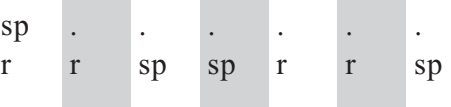

$750-1200$

$100-1300$

$250-450$

800

$\mathrm{u}$

$\mathrm{sp}$ $\mathrm{sp}$
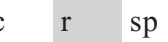

$r$

[u]

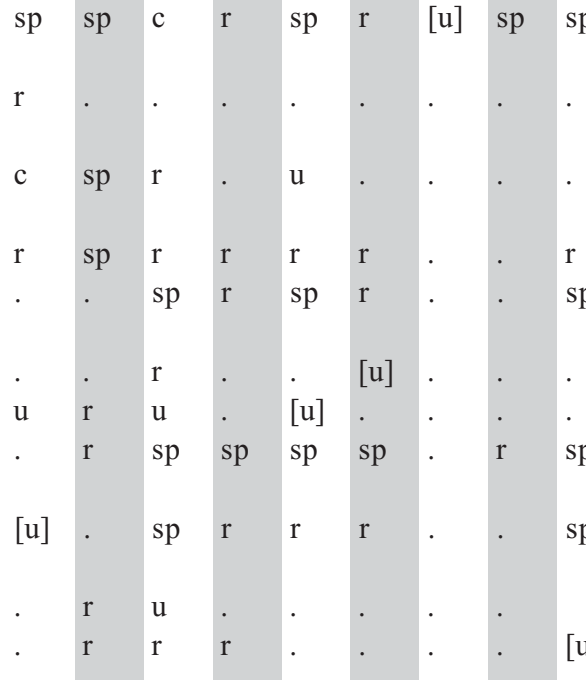

$1200-140$

200-1250

100-600 + ++

sp $100-350+++$

200

250-750

$150-400$

p $100-750$

300-550

[u] $200-450$

370

$\mathrm{sp}$

250-1400

100-300

$1000-1400$

$100-200$

$100-250$

$100-600$

300

100-200

400

300

500-950

[u] [u]
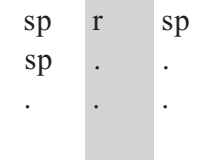

r

$\mathrm{r} \quad[\mathrm{u}]$

[u]

Total number of species in regions

$\begin{array}{lllllll}302 & 232 & 235 & 148 & 233 & 170 & 82\end{array}$ 


\section{COMMENTS}

For species with a single record within relevant geographic regions, locations and dates of collection by the author are given. In case the location was published in Arctoa, only bibliography is cited.

1. Abietinella abietina - KS: Ust'-Kormikha, 28.VI.2010. VP: Kishtovka, 10.VII.2009.

2. Acaulon triquetrum - UO: (Pisarenko, 2012a).

3. Aloina brevirostris - SR: Aprel'ka (Pisarenko, 2007a). TR: Tomsk (Muldiyarov et al., 2013).

4. Amblystegium radicale-KA: Akchelbak creek (Muldiyarov \& Lapshina, 1996).

5. Amphidium mougeotii - KA: Rastai River, 30.VI.2005.

6. Anacamptodon latidens - KA: (Pisarenko, 2004).

7. Anomodon attenuatus - TR: (Pisarenko, 2007a).

8. A. longifolius - TR: Izdrevaja River, 30.V.2008. UO: Chaschino, 11.VI.2011.

9. A. viticulosus - TR: Bugotak River, 26.IV.2009. UO: Chaschino, 11.VI.2011.

10. Atrichum tenellum - from literature data only. SM: Kuzedeevo (Gudoshnikov, 1986). TR: (Lapshina \& Muldiyarov, 1998).

11. A. undulatum - UO: Acutikha, 14.VII.2007. Well developed plants with sporophytes often occur as admixture to $A$. flavisetum.

12. Barbula convoluta - from literature data only. TR: (Lapshina \& Muldiyarov, 1998). UO: Kolyvan“ (Dyachenko, Taran, 2012).

13. Bartramia pomiformis - SR: Egorievsk, 13.VIII.1990. TR: Mariinsk, 8.VI.2007.

14. Brachytheciastrum velutinum - UO: Ovechkino, Kontoshino, Rasskaziha, Sosnovka, Barnaul (Nozhinkov, 2004, 2006), and Bistry Istok (Nozhinkov \& Taran, 2008).

15. Brachythecium is treated according to Ignatov \& Milyutina (2010), Ignatov (2012).

16. Brachythecium albicans - SM: SM: Kuzedeevo (Gudoshnikov, 1986). SR: Kotorovo (Gudoshnikov, 1986).

17. B. baicalense - SR: Apanas, 8.VI.2008. UO: Erestnaja, 9.IX.2011. Identified by M.S. Ignatov.

18. B. campestre-SM: SM: Kuzedeevo (Gudoshnikov, 1986).

19. B. complanatum - SM: SM: (Pisarenko, 2013).

20. B. erythrorrhizon - VP: vicinity of Igol (Lapshina \& Muldiyarov, 1998).

21. Breidleria pratensis - KA: Azhendarovsky ridge, Inyushka, 10.VI.2010.

22. Bryobrittonia longipes - KA: Makarakskiy Creek (Vasiliev, 1974).

23. Bryum treated according to Zolotov (2011).

24. Bryum capillare - TR: (Lapshina \& Muldiyarov, 1998).

25. B. cyclophyllum - TR: (Ignatova \& Pisarenko, 2013a).

26. B. elegans - KS: Malinovoe Lake and Volchikha (Nozhinkov, 2006). UO: Barnaul (Nozhinkov, 2004), Sosnovy island (Nozhinkov \& Taran, 2008).

27. B. funkii-TR: Serta River bank, 7.06.2007. UO: Beloglazovo (Nozhinkov, 2006).

28. B. intermedium - SR: Suenga River in Kemenka Creek mouth (Pisarenko, 2007b). VP: (Lapshina \& Muldiyarov, 1998).

29. B. moravicum - UO: some points in Ob islands and floodplain Bistry Istok (Nozhinkov \& Taran, 2008).

30. B. neodamense - SR: Krasnoye, 22.VI.1994. (Pisarenko, 2007b).

31. B. pallens - TR: (Lapshina \& Muldiyarov, 1998). UO:
Niznekamenka, 10.IX.2012.

32. B. pallescens - KA: Stanovoj ridge (Volkova \& Muldiyarov, 2000). Vp\&, TR: (Lapshina \& Muldiyarov, 1998). The records are doubtfull and possibly reffer to $B$. lonchocaulon (Zolotov, 2000, 2011).

33. B. subapiculatum - UO: Kontoshino (Nozinkov, 2006).

34. B. weigelii - VP: Melnikovo (Muldiyarov et al., 2013)

35. Buxbaumia aphylla - TR: Tomsk (Krylov, 1924). The only record in study area; never collected later.

36. Calliergon giganteum - UO: Sosnovka (Nozhinkov, 2006).

37. C. megalophyllum - was mentioned for Igol surroundings (Lapshina \& Muldiyarov, 1998), but late was not listed (Lapshina, 2003).

38. Campyliadelphus chrysophyllus - UO: some points in $\mathrm{Ob}$ islands and floodplain Bistry Istok (Nozhinkov \& Taran, 2008).

39. Campylidium calcareum - SR: Suenga River (Pisarenko, 2007b).

40. Campylophyllum halleri - KA: (Pisarenko, 2004).

41. Cinclidium stygium - SR: Krasnoye (Pisarenko, 2007b). TR: (Pisarenko, 2007k).

42. Conardia compacta - KS: Volchiha (Nozhinkov, 2004). UO: Sosnovy island on Ob River (Nozhinkov \& Taran, 2008).

43. Cratoneuron filicinum - VP: (Lapshina \& Muldiyarov, 1998).

44. Cynodontium tenellum - SR: Novososedovo (Pisarenko, 2007b)

45. Dichelyma falcatum - KA: (Pisarenko, 2004).

46. Dichodontium pellucidum - SR: Udinsk (Pisarenko, 2007b). TR: (Muldiyarov \& Chernova, 2002).

47. Dicranella crispa - TR: (Lapshina \& Muldiyarov, 1998).

UO: Beloglazovo (Nozhinkov, 2006).

48. D. schreberiana - VP: (Lapshina \& Muldiyarov, 1998).

49. D. subulata - TR: (Lapshina \& Muldiyarov, 1998). UO: Beloglazovo (Nozhinkov, 2006).

50. D. varia - TR: (Muldiyarov \& Chernova, 2002).

51. Dicranum acutifolium - TR: Tambar, 6.VI.2007. KS: Volchiha (Nozhinkov, 2004).

52. D. brevifolium - UO: Klochki (Nozhinkov, 2004).

53. D. drummondii - UO: Pankrushiha (Nozhinkov, 2004).

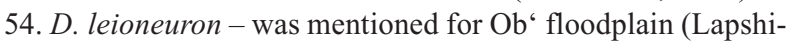
na \& Muldiyarov, 1998), but later not listed (Lapshina, 2003). 55. D. viride - SR: (Ignatova, Fedosov, 2008).

56. Didymodon ferrugineus - TR: (Muldiyarov \& Chernova, 2002).

57. D. icmadophilus - TR: (Lapshina \& Muldiyarov, 1998).

UO: Beloglazovo (Nozhinkov, 2006).

58. D. tophaceus - TR: (Muldiyarov \& Chernova, 2002).

59. Distichium inclinatum - VP: Desjatovo (Lapshina \& Muldiyarov, 1998).

60. Ditrichum cylindricum - TR: (Lapshina \& Muldiyarov, 1998). UO: Beloglazovo (Nozhinkov, 2006).

61. D. heteromallum - TR: (Lapshina \& Muldiyarov, 1998).

UO: Beloglazovo (Nozhinkov, 2006).

62. D. pusillum - KA: Kija River valley near Bezimianka Creek, 4.VII.2005.

63. Drepanocladus sendtneri - SR: Krasnoye (Pisarenko, 2007b). TR: Tenguli, 13.VI.2008. KD: Pushkino, 31.V.2011. 64. Encalypta treated according to Fedosov $(2012,2013)$.

65. Encalypta rhaptocarpa - KA: N.Tres' River valley, 30.VIII.2003.

66. E. trachymitria - SM: Ust-Kobirza, 28.VIII.2011.

67. E. vulgaris - VP: vicinity of Kishtovka, 10.VII.2009. 
68. Entodon concinnus - KA: (Pisarenko, 2004). SR: Kostenkovo (Pisarenko, 2007b).

69. E. schleicheri-KD: Kalachevo, 16.VI.2007.

70. Fabronia ciliaris Brid. - SR: Pecherkino (Pisarenko, 2007b).

71. Fissidens adianthoides - UO: Novokamenka, 10.IX.2011. VP: (Lapshina \& Muldiyarov, 1998).

72. Fissidens exilis - KD: Kljuchi, 10.VI.2010.

73. Fissidens osmundoides - SR: M.Chumish River (Pisarenko, 2007b).

74. F. taxifolius - UO: some points in Ob islands and floodplain Bistry Istok (Nozhinkov \& Taran, 2008).

75. F. viridulus - mentioned in literature - TR, VP: (Lapshina \& Muldiyarov, 1998). I didn't try to distinguish it from $F$. bryoides according to Ignatov \& Ignatova (2003).

76. Fontinalis hypnoides - UO: Rasskazikha (Nozhinkov, 2006); between settlements Ust'-Anuj and Bistry Istok (Nozhinkov \& Taran, 2008)

77. Grimmia anodon - SM: Ust-Anzas, 3.VIII.2010. KD: (Pisarenko, 2007).

78. G. funalis - SM: Pustag Mt., 1.VIII.2010.

79. Grimmia pulvinata - SM: Podkatun (Nozhinkov \& Pisarenko, 2008; by Nozhinkov data only).

80. G. tergestina - TR: Krylovo, 13.VI.2007.

81. G. unicolor - KA: Barkhatnij ridge, 16.VI.2000.

82. Gymnostomum aeruginosum - TR: (Pisarenko 2012c).

83. Hamatocaulis vernicosus - KA: Poludnevaja creek (Volkova \& Muldiyarov, 2000). UO: (Pisarenko et al., 2007).

84. Haplocladium angustifolium - KA: (Pisarenko, 2004).

85. Helodium blandowii - KS: Vavilon Lake (Pisarenko et al., 2008).

86. Herzogiella striatella - KA: (Pisarenko, 2004).

87. H. turfacea - SM: Ust‘ Anzas (Nozhinkov \& Pisarenko, 2008).

88. Heterocladium dimorphum - TR: Bogashevo and Tugoiakovka River (Muldiyarov \& Lapshina,1998)

89. Homalia trichomanoides - UO: Chatchino, 11.VI.2011.

90. Hygroamblystegium humile - KA: Kija River valley (Volkova \& Muldiyarov, 2000).

91. Hygroamblystegium tenax - TR: Tomsk (Lapshina \& Muldiyarov, 1998). The record is doubtful.

92. Hypnum cupressiforme - VP: (Lapshina \& Muldiyarov, 1998). KS: Volchicha (Nozhinkov, 2004). UO: Sosnovka (Nozhinkov, 2006).

93. Isopterygiopsis alpicola-KA: Chemodan Mt., 11.IX.2004.

94. I. muelleriana - KA: Chemodan Mt., 11.IX.2004.

95. I. pulchella - UO: Bistry Istok (Nozhinkov \& Taran, 2008).

96. Iwatsukiella leucotricha-SM: Pustag Mt., 27.IV.2010.

97. Jaffueliobryum latifolium - KS: (Pisarenko, Ignatova, Ignatov, 2001).

98. Lescuraea incurvata - VP: Barkovsky i. (Muldiyarov et al., 1998)

99. Leucodon sciuroides Schwagr. - TR: (Pisarenko, 2012c). KD: Krylovo, 13.VI.2007.

100. Meesia triquetra - KA: Krestovka River (Volkova \& Muldiyarov, 2000). TR: Tambar, 6.VI.2007. UO: (Pisarenko et al., 2007).

101. Meesia uliginosa - KA: Chemodan Mt., VII.1993, coll. N.Lashchinsky (Pisarenko, 2009)

102. Microbryum curvicollum - UO: (Pisarenko, 2012a)

103. Mnium spinulosum - SM: Ust'-Anzas (Nozinkov \& Pisarenko, 2008). TR: (Pisarenko, 2012c).
104. Myurella julacea - KA: (Pisarenko, 2004).

105. M. tenerrima - KA: (Pisarenko, 2004). TR: Ust'-Kolba, 7.VI.2007.

106. Niphotrichum canescens - TR: (Muldiyarov \& Chernova, 2002)

107. Oncophorus virens - VP: (Lapshina \& Muldiyarov, 1998).

108. Orthothecium intricatum - KA: (Pisarenko, 2004).

109. Orthotrichum alpestre - SR: Suenga River near Kamenka mouth (Pisarenko, 2007b).

110. O. pallens - SR: Sungai (Pisarenko, 2007b).

111. O. pellucidum - SR: Novososedovo (Pisarenko, 2007b).

112. O. rupestre - SM: Podkatun (Nozhinkov \& Pisarenko, 2008). SR: Suenga River near Kamenka mouth (Pisarenko, 2007b).

113. Oxyrrhynchium hians - UO: Barnaul and Kontoshino (Nozhinkov, 2006); some points in Ob islands and floodplain Bistry Istok (Nozhinkov \& Taran, 2008).

114. Paludella squarrosa - TR: Tambar, 6.VI.2007. UO: (Pisarenko et al., 2007).

115. Palustriella commutata - TR: (Lapshina \& Muldiyarov, 1998).

116. P. falcata - SR: Krasnoje, 9.VI.2008.

117. Paraleucobryum enerve - KA: (Pisarenko, 2004).

118. Paraleucobryum longifolium - TR: Mariinsk, 8.VI.2007.

119. Philonotis is treated according to Koponen \& al (2012).

120. Philonotis fontana - SR: Novososedovo (Pisarenko, 2007b). UO: Velizhanovka (Nozhinkov, 2004)

121. Physcomitrella patens - TR: Inja River Novosibirsk, coll. Taran, 2001. BS: Kreschenskoje, 18.VII.2012. UO: some points in $\mathrm{Ob}$ islands and floodplain Bistry Istok (Nozhinkov \& Taran, 2008).

122. Physcomitrium eurystomum - UO: between s Ust'-Anuj and Bistry Istok (Nozhinkov \& Taran, 2008).

123. P. pyriforme - TR: Tambar, 6.VI.2007. UO: Barnaul (Nozhinkov, 2006).

124. Plagiomnium affine - SM: Pyzas River Shanyshtag Mt., 19.VII.2007.

125. P. confertidens - UO: Roznev Log (Nozhinkov, 2004).

126. P. drummondii - UO: Krasilovo Lake; Bobrovka and Firsovo s (Nozhinkov, 2006).

127. P. rostratum - VP: (Lapshina \& Muldiyarov, 1998).

128. Plagiopus oederianus - TR: Izdrevaja River, 18.XI.2004.

129. Plagiothecium cavifolium - TR: Mariinsk, 8.VI.2007.

130. P. laetum - UO: Bistry Istok (Nozhinkov \& Taran, 2008).

131. P. latebricola - TR: Troitskoje, 15.VI.2010.

132. Platydictya jungermannioides - KA: (Pisarenko, 2004) SM: Kuzedeevo (Gudoshnikov, 1986).VP: Ponkino (Pisarenko et al., 2011).

133. Pleuridium subulatum - TR: Gorniy, 17.VI.2010. KS: (Pisarenko et al., 2001).

134. Podperaea krylovii - SR: Suenga River near Kamenka mouth (Pisarenko, 2007b).

135. Pogonatum urnigerum - TR: (Lapshina \& Muldiyarov, 1998). VP: Kyshtovka, 10.VII.2009.

136. Pohlia annotina - SM: Ust' Kobyrza (Nozhinkov, Pisarenko, 2008). UO: Bistry Istok (Nozhinkov \& Taran, 2008).

137. P. elongata - TR: Mariinsk, 8.VI.2007.

138. P. longicollis - KA: Rastai River (Pisarenko, 2009). SM: Pyzas River, 19.VII.2007.

139. P. melanodon - TR: (Lapshina \& Muldiyarov, 1998). KD: Morkovkino, 10.VI.2009. UO: Barnaul (Nozhinkov, 2006). 140. P. obtusifolia - KA: (Pisarenko, 2004). 
141. P. sphagnicola - KA: Chemodan Mt. (Muldiyarov \& Lapshina, 1996). TR: Tomsk (Lapshina \& Muldiyarov, 1998). VP: (Pisarenko et al., 2011; by Muldiyarov data only).

142. Polytrichastrum alpinum - SR: Eltsovka (Pisarenko, 2007b).

143. P. formosum - KS: Volchiha (Nozhinkov, 2004). UO: Barnaul, Podbornoje (Nozhinkov, 2004).

144. P. longisetum - SR: Bol'shie Taily River (Pisarenko, 2007b). KD: Bannovo, 15.VI.2009.

145. P. pallidisetum - KA: (Pisarenko, 2004).

146. Polytrichum commune - BS: Orlovskiy (Valutskiy, 2011)

147. P. strictum - KS: Volchiha (Nozhinkov, 2004). UO: Barnaul (Nozhinkov, 2004).

148. Pseudephemerum nitidum - KA: (Pisarenko, 2004).

149. Pseudocalliergon trifarium - KA: Chemodan Mt. (Volkova \& Muldiyarov, 2000). SR: Krasnoye (Pisarenko, 2007b).

150. Pseudoleskeella nervosa - UO: Beloyarsk (Nozhinkov, 2006).

151. P. papillosa - SR: M.Ik River (Pisarenko, 2007b).

152. P. rupestris - SR: Koltyrak, 4.VI.1992.

153. Pterigynandrum filiforme - SR: Kivda Mt. (Pisarenko, 2007b).

154. Pterygoneurum subsessile - SR: Suenga (Pisarenko, 2007b). KD: Artyshna, 17.VI.2007.

155. Rhizomnium andrewsianum - SM: Ust'-Kobirza, 29.VIII.2011.

156. R. punctatum - TR: (Lapshina \& Muldiyarov, 1998). UO: Semenovskij, 10.VI.2011.

157. Rhodobryum ontariense - TR, KD: (Pisarenko, 2007a).

158. Rhynchostegium rotundifolium - KA: Saltimakov ridge (Pisarenko, 2009). SM: watershed Taenza and Mrassu Rivers, 4.VIII.2010.

159. Rhytidiadelphus subpinnatus - TR: (Lapshina \& Muldiyarov, 1998). UO: Yasnaja Poliana, 30.VI.2010.

160. Rhytidium rugosum - UO: Verkh-Ozeronje (Nozhinkov

\& Taran, 2008). VP: Kyshtovka, 10.VII.2009.

161. Schistidium is treated according to Ignatova (2012).

162. Schistidium apocarpum - UO: Sosnovy island (Nozhinkov \& Taran, 2008).

163. S. obscurum - SM: (Ignatova \& Pisarenko, 2013b). 164. S. platyphyllum - TR: (Ignatova \& Pisarenko, 2013a).

165. S. rivulare - TR: (Muldiyarov \& Chernova, 2002)

166. S. sibiricum - TR: (Ignatova \& Pisarenko, 2013b).

167. S. sinensiapocarpum - TR: (Ignatova \& Pisarenko, 2013b).

168. S. submuticum - TR: (Ignatova \& Pisarenko, 2013b).

169. S. tenuinerve - KD: (Ignatova \& Pisarenko, 2013a).

170. Schistostega pennata - SR: Kotorovo (Pisarenko, 2007b).

KD: Luchshevo, 11.VI.2010.

171. Sciuro-hypnum plumosum - KS: Volchiha (Nozhinkov, 2004).

172. Scorpidium cossonii - KA: Chemodan Mt. (Muldiyarov \& Lapshina, 1996). SR: Krasnoye (Pisarenko, 2007b).

173. S. revolvens - KA: Chemodan Mt. (Volkova \& Muldiyarov, 2000)

174. Seligeria brevifolia - KA: Ters` River, 30.VIII.2003.

175. Seligeria campylopoda - TR: (Muldiyarov \& Chernova, 2002).

176. S. donniana-SM: Ust` Kobyrza (Nozhinkov\&Pisarenko, 2008).

177. S. pusilla - KA: (Pisarenko, 2004). SM: Ust‘ Kobyrza (Nozhinkov \& Pisarenko, 2008).

178. S. tristichoides - KA: (Pisarenko, 2004). SM: Ust‘ Kob- yrza (Nozhinkov \& Pisarenko, 2008).

179. Serpoleskea subtilis - KS: Volchiha (Nozhinkov, 2004).

180. Sphagnum aongstroemii - KA: Chemodan Mt. (Muldiyarov \& Lapshina, 1996).

181. S. balticum - KA: Chemodan Mt. (Muldiyarov \& Lapshina, 1996). TR: Tomsk (Lapshina \& Muldiyarov, 1998). UO: Verhnij Suzun River, Osinovoe Lake, 1997.

182. S. centrale - KA: Chemodan Mt. (Muldiyarov \& Lapshina, 1996). KS: Volchiha (Nozhinkov, 2004).

183. S. compactum - TR: (Lapshina \& Muldiyarov, 1998).

184. S. contortum - KA: Chemodan Mt. (Muldiyarov \& Lapshina, 1996). UO: Larichikha, 18.VIII.2013.

185. S. cuspidatum - VP: Uzas, by Muldiyarov data (Pisarenko et al., 2011).

186. S. fallax - KS: Volchiha (Nozhinkov, 2004).

187. S. fimbriatum- KD: Novobarachaty, 3.VI.2011.

188. S. flexuosum - TR: (Lapshina \& Muldiyarov, 1998).

189. S. girgensohnii - KS: Volchiha (Nozhinkov, 2004). UO: Barnaul (Nozhinkov, 2004).

190. S. inundatum - SR: Mirny (Pisarenko, 2007b).

191. S. jensenii - SR: Mirny (Pisarenko, 2007b).

192. S. majus - KA: Chemodan Mt. (Muldiyarov \& Lapshina, 1996). SR: Mirny (Pisarenko, 2007b). TR: (Lapshina \& Muldiyarov, 1998).

193. S. obtusum - KA: Chemodan Mt. (Muldiyarov \& Lapshina, 1996)

194. S. palustre - KA: Chemodan Mt. (Muldiyarov \& Lapshina, 1996). SR: Mokrushino (Pisarenko, 2007b). TR: (Lapshina \& Muldiyarov, 1998).

195. S. platyphyllum - UO: Ryamy, coll. S.Vasivl'ev, 1997; Pisarenko 18.VIII.2013.

196. S. pulchrum - KA: Poludnevaja creek (Muldiyarov \& Lapshina, 1996).VP: Targach Lake (Pisarenko et al., 2011).

197. S. riparium - TR: (Lapshina \& Muldiyarov, 1998). VP: (Pisarenko et al., 2007).

198. S. rubellum - BS: between Sherstobitovo and Filimonovo settlements (Valutskiy, 2011).

199. S. squarrosum - KS: Vavilon Lake, (Pisarenko et al., 2008).

200. S. subfulvum - VP: Uzas, by Muldiyarov data (Pisarenko et al., 2011).

201. S. subnitens - KA: Chemodan Mt. (Muldiyarov \& Lapshina, 1996).

202. S. tenellum - KA: Chemodan Mt. (Muldiyarov \& Lapshina, 1996).

203. S. teres - KA: Poludnevaja creek; Chemodan Mt. (Muldiyarov \& Lapshina, 1996). KD: between Berezovka and Bannovo s, 16.VI.2009. KS: Vavilon Lake (Pisarenko et al., 2007).

204. S. wulfianum - KA: Azhendarovsky ridge, Klyuchi, 8.VI.2010. TR: (Lapshina \& Muldiyarov, 1998).

205. Splachnum ampullaceum - VP: Targach Lake (Pisarenko et al., 2011).

206. Straminergon stramineum - TR: (Lapshina \& Muldiyarov, 1998).

207. Syntrichia ruralis - VP: Kyshtovka, 10.VII.2009.

208. Tayloria lingulata - KA: (Pisarenko, 2004).

209. Tetraphis pellucida - KS: Volchiha (Nozhinkov, 2004).

210. Thamnobryum neckeroides - TR: Mariinsk, 8.VI.2007.

211. Timmia bavarica - UO: Sosnovka (Nozhinkov, 2006).

212. T. comata - KA: (Pisarenko, 2004). SR: Kostenkovo (Pisarenko, 2007b). TR: (Muldiyarov \& Chernova, 2002). 
213. Tomentypnum nitens - KA: Kija River valley (Volkova \& Muldiyarov, 2000).

214. Tortella alpicola - SR: Kostenkovo, 24.VI.1994.

215. T. fragilis - VP: Desjatovo (Lapshina \& Muldiyarov, 1998).

216. T. inclinata - KD: (Pisarenko, 2007a).

217. Tortula acaulon - TR: Gorny, 17.VI.2010. UO: VerkhOzeronje (Nozhinkov \& Taran, 2008).

218. T. muralis - SM: vicinity of Kuzedeevo, data of A.N. Vasiljev, 14.IX.1970. SR: Novososedovo (Pisarenko, 2007b). KS: Blagoveschenka, 26.VI.2010.

219. Tortula obtusifolia - SM: Podkatun, data of Nozhinkov (Nozhinkov \& Pisarenko, 2008).

220. Trachycystis ussuriensis - SM: (Pisarenko, 2012a).

221. Trichostomum crispulum - SM: Ust'-Anzas Settelment, 3.VIII.2010.

222. Ulota rehmannii Jur. - SM: Sheregesh, 27.IV.2010.

223. Warnstorfia exannulata - TR: (Lapshina \& Muldiyarov, 1998). UO: Barnaul (Nozhinkov, 2006).

224. Warnstorfia fluitans - UO: Barnaul (Nozhinkov, 2006).

225. Weissia brachycarpa - UO: Verkh-Ozeronje (Nozhinkov \& Taran, 2008).

226. W. controversa-SM: Ust' Kobyrza, according to Nozhinkov (Nozhinkov\&Pisarenko, 2008). TR: (Lapshina \& Muldiyarov, 1998).

227. W. longifolia - TR: (Pisarenko 2012c).

228. Zygodon sibiricus -VP: (Pisarenko, 2012c).

\section{DISCUSSION}

By now, 425 species of mosses are known from the study territory.

The maximum species diversity is associated with mountains of Kuznetskiy Alatau and Mountain Shoria. Overall 342 species are recorded for these two regions, and 85 of them were not found in other regions of the investigated territory. Currently less species are recorded for Mountain Shoria than for Kuznetskiy Alatau (232 vs.302), which can be explained partly by a better exploration of the latter.

Bryoflora of Salair Ridge is significantly poorer than of Kuznetsk Alatau: 235 vs. 302. Of course, Salair is a low mountain formation and has no alpine or mountain tundra belts. However, the absence of a number species in the forest belt is notable. A lower diversity of the Salair bryoflora is likely related to low air humidity, which reduces the number of species almost down to the number of that in transitional region (233 species).

Kulunda is poorer in species number (82), because of xeric and windy climate, flat area and strong antropogenic disturbance. The least species are recorded for Baraba (65); but in this case the low number is likely explained by insufficient investigations.

Kuznetskaya depression, upper $\mathrm{Ob}$ and Vasyugan plain with adjacent areas are almost identical in mosses number (148, 170, and 169 species), but not in composition.

Vasyugan region stands out by a group of species concentrated in its northern part. These are mainly mire species which are common in Vasyugan plain and northwards; only few of them penetrate to the forest-steppe and steppe zones of West Siberia. Thus, only half of Sphagnum species from the list participate in mire communities of plains southwards of Vasyugan: Sphagnum angustifolium, S. capillifolium, S. centrale, S. fimbriatum, S. fuscum, S. magellanicum, S. russowii, S. squarrosum, $S$. teres, $S$. warnstorfii. At the same time, Vasyugan plain represents the southern boundary (within the West Siberian longitudinal sector) for Sphagnum lindbergii, S. obtusum, S. papillosum, S. palustre, S. riparium, $S$. rubellum, as well as for Bryum neodamense, Calliergon richardsonii, Cinclidium stygium, Drepanocladus sendtneri, Hamatocaulis lapponicus, H. vernicosus, Meesia longiseta, M. triquetra, M. uliginosa, Pseudocalliergon lycopodioides, Pseudocalliergon trifarium, Scorpidium scorpioides. Some of these species have scattered records in forest-steppe and steppe zones, in mires with a number of relicts of the last glacial period.

Xeric species in the study area are centered in southern depressions and plains (Kulunda, upper Ob, transitional region, Kuznetskaya depression); most common are Encalypta vulgaris, Jaffueliobryum latifolium, Pterygoneurum ovatum, $P$. subsessile, Tortula acaulon, $T$. mucronifolia. All of them, with the exception only for $P$. ovatum, were also recorded in the petrophyte steppe of Salair, where additionally the following species occur: Aloina brevirostris, Fabronia ciliaris, Grimmia laevigata, Homomallium incurvatum. This group becomes poorly represented in Mountain Shoria region, where only Homomallium incurvatum and Grimmia anodon were recorded. No one of the above mentioned species has been recorded in Kuznetskiy Alatau, where such widespread species as Syntrichia ruralis and Hedwigia ciliata are very rare. Some more xeric species, which are rare in the study area include: Aloina rigida, Grimmia tergestina, Tortula modica, Weissia longifolia (transitional region); Acaulon triquetrum, Microbryum curvicollum (upper $\mathrm{Ob}$ ); Entosthodon hungaricus (Kulunda); Conardia compacta, Pterygoneurum kozlovii (Kulunda \& upper $\mathrm{Ob}$ ).

Diversity and specificity of combined bryoflora of Kuznetskiy Alatau and Mountain Shoria depends to a great extent on the presence of mountain and alpine species, e.g., Andreaea rupestris, Aulacomnium turgidum, Bartramia ithyphylla, Brachythecium erythrorrhizon, Dicranodontium denudatum, Dicranum elongatum, D. spadiceum, Drepanium recurvatum, Kiaeria starkei, Oligotrichum hercynicum, Polytrichastrum alpinum, Polytrichastrum sexangulare.

Petrophytic species are also almost exclusively concentrated in Kuznetsk Alatau and Mountain Shoria (Hymenoloma crispulum, Lescuraea saxicola, L. incurvata, Bucklandiella microcarpa, B. sudetica, Grimmia spp., Schistidium spp., and other). In the investigated area, thick Quaternary sediments are expressed almost throughout. Outside the mountains of Kuznetsk Alatau, Mountain Shoria and Salair, rock outcrops are extremely rare 
and are confined to river valleys or steep slopes of the hills. Low humidity in the plains within subtaiga-steppe zones is a limiting factor for most petrophytes, along with the rarity of suitable habitats. Most common petrophytes are Didymodon validus s.l., Distichium capillaceum, Encalypta pilifera, E. vulgaris, Grimmia longirostris, Hedwigia ciliata, Myurella sibirica, Orthotrichum anomalum, Pseudoleskeella tectorum, Saelania glaucescens, Schistidium lancifolium, S. pulchrum, Sciuro-hypnum populeum, Tortella tortuosa, Syntrichia ruralis. Many species commonly growing as epilithic in mountains occur in other habitats in lowlands: (1) Distichium capillaceum, D. inclinatum, Saelania glaucescens in Tomsk and northern part of Novosibirsk Province grow on degradated peat in mire massifs; (2) Abietinella abietina, Encalypta vulgaris, Rhytidium rugosum, Syntrichia ruralis were collected on edge of a steep bank of the Tara River (in vicinity of Kishtovka Settlement); (3) Anomodon longifolius was collected on Picea-trunks in Inia River valley (vicinity of Chaschino Settlement), Leucodon sciuroides and Rhytidium rugosum - on Betula-trunks in mesophytic forests near Novosibirsk.

Mosses of the forest belt contribute to specificity of bryoflora of Kuznetsk highland besides the alpine (arcto-alpine) and petrophytic species. Thus, Eurhynchium angustirete, Hylocomiastrum umbratum, Sciuro-hypnum ornellanum are rather common and abundant in the ground cover of Abies-forests in Kuznetskiy Alatau and Mountain Shoria, being absent not only in lowland bryoflora, but also in rather similar Abies-forests of Salair. The same distributional pattern occurs for epiphytic and epixylic Ulota rehmannii, Zygodon sibiricus, Iwatsukiella leucotricha Anacamptodon latidens. Moreover, some hydrophytes Cinclidotus riparius, Fontinalis hypnoides, Dichelyma falcatum, Palustriella decipiens, Bryum schleicheri occur in and near streams in the forest belt of Kuznetsk Alatau and/or Mountain Shoria; but were never found outside the region within the study area. The same is true for calciphilous epilitic mosses including Brachythecium cirrosum, Bryobrittonia longipes, Campylophyllum halleri, Cyrtomnium hymenophylloides, Mnium thomsonii, Orthothecium intricatum.

Most species are widespread in Holarctic. Only some species have mainly western distribution (Acaulon triquetrum, Dicranum viride, Entosthodon hungaricus, Herzogiella striatella, Heterocladium dimorphum, Pleuridium subulatum, Taxiphyllum wissgrillii); some - mainly eastern distribution (Anacamptodon latidens, Iwatsukiella leucotricha, Jaffueliobryum latifolium, Myuroclada maximowiczii, Podperaea krylovii, Zygodon sibiricus), while some are common in the eastern part of Russia, penetrating to European Russia only in the Caucasus and Urals: Fabronia ciliaris, Rhodobryum ontariense, Trachycystis ussuriensis.

Most interesting rare species for the territory are: Acaulon triquetrum, Cnestrum schistii, Conardia compacta, Dicranum viride, Entosthodon hungaricus, Grimmia anomala, Herzogiella striatella, Isopterygiopsis alpicola, Microbryum curvicollum, Oligotrichum hercynicum, Pleuridium subulatum, Podperaea krylovii, Pohlia obtusifolia, Polytrichastrum pallidisetum, Pseudephemerum nitidum, Pseudocalliergon lycopodioides, Rhynchostegium arcticum, Rhynchostegium rotundifolium, Taxiphyllum wissgrillii, Tortella alpicola, and Tayloria lingulata. All of them are known in Siberia from scattered localities.

\section{ACKNOWLEDGEMENTS}

I was very happy to have dozens of cooperative expeditions with N.N. Lashchinsky, A.Yu. Korolyuk, E.D. Lapshina, E.Ya. Muldiyarov, N.I. Makunina; I am grateful to these people for joint work and comments about Siberian vegetation.

\section{LITERATURE CITED}

[CHEREPANOV, S.K.] ЧЕРЕПАНОВ С.К. 1995. Сосудистые растения России и сопредельных государств (в пределах бывшего CCCP). - Vascular plants of Russia and adjacent states (the former USSR)] СПб [St.-Peterburg]. $992 \mathrm{pp}$.

[DYACHENKO, A.P. \& G.S. TARAN] ДЬЯЧЕНКО А.П., Г.С. ТАРАН 2012а. К бриофлоре пойменных лесов реки Оби в подзоне северной лесостепи. - [On the Ob River floodplain forests bryoflora in the norther forest-steppr subzone] Вестник ТГУ. Биология [Vestnik Tomsk. Gos. Uni Ser. Biol.] 4(20): 93-104.

[DYACHENKO, A.P. \& G.S. TARAN] ДЬЯЧЕНКО А.П., Г.С. ТАРАН 2012 b. Новые находки мхов в Новосибирской области. 1. - [New moss records from Novosibirsk Province. 1] Arctoa 21: 289.

FEDOSOV, V.E. 2012. Encalypta sect. Rhabdotheca in Russia. - Arctoa 21: $101-112$.

[FEDOSOV, V.E.] ФЕДОСОВ В.Э. 2013. Encalypta Hedw. - Флора мхов России, России [Moss flora of Russia] http://arctoa.ru/Flora/taxonomy-ru/taxonomy-ru.php (версия 20.II.2013).

[FINER, YU.B.] ФАЙНЕР Ю.Б. 1969. Кузнецкая котловина. - [Kuznetsk depression] В кн.: Стрелков С.А., В.В. Вдовин (ред.) История развития рельефа Сибири и Дальнего Востока. Алтае-Саянская горная область. M., Наука [In: Strelkov, S.A. \& V.V. Vdovin (eds.) Istorija razvitija reliefa Sibiri i Dalnego Vostoka. Altaie-Sajanskaja gornaja oblast. Moscow, Nauka]: 157-203.

[GORODETSKAYA, M.E.] ГОРОДЕЦКАЯ М.Е. 1975. Западная Сибирь. Основные черты орографии. - [West Siberia. The main features of the orography] В кн.: Коржуев С.С. (ред.) Равнины и горы Сибири. М.: Наука [In: Korzhuev, S.S. (ed.) Plains and mountains of Siberia. Moskow, Nauka]: 10-12.

[GORODETSKAYA, M.E. \& G.I LAZUKOV] ГОРОДЕЦКАЯ М.E., Г.И. ЛАЗУКОВ 1975. Западная Сибирь. Геоморфологическое районирование. - [West Siberia. Geomorphological zoning] В кн.: Коржуев С.С. (ред.) Равнины и горы Сибири. М., Наука [In: Коrzhuev, S.S. (ed.) Plains and mountains of Siberia. Moskow, Nauka]: 118-121.

[GORODKOV, B.N.] ГОРОДКОВ Б.Н. 1915. Подзона лиственных лесов в пределах Ишимского уезда Тобольской губернии. - [Deciduous forest subzone within Ishim uezd of Tobolsk Province] $B$ кн.: Федченко Б.А. (ред.) Труды почв.-ботан. Экспедиций Переселенческого управления по исследованию колониз. Районов Азиатской России в 1912 г. Петроград. [In: Fedchenko B.A. (ed.) Trudy pochv.bot. Ekspeditsy Pereselencheskogo upravleniya po issledovaniyu coloniz. Rajonov Aziatskoy Russia. Petrograd] 2 (3): 102-133.

[GRITSENKO, A.G. \& V.A. NIKOLAEV] ГРИЦЕНКО А.Г., В.А. НИКОЛАЕВ 1988. Морфометрия рельефа южных равнин Западной Сибири. - [Morphometry of relief of West Siberia southern plains] В кн.: Кашменская О.В. (ред.) Рельеф Западно-Сибирской равниныл. Новосибирск, Наука [In: Kashmenskaya, O.V. (ed.) Relief Zapadno-Sibirskoi ravnini. Novosibirsk, Nauka]: 139-145. 
[GUDOSHNIKOV, S.V.] ГУДОШНИКОВ С.В. 1976. Флора листостебельных мхов Кузедеевского липового острова в предгорьях Кузнецкого Алатау. - [The flora of leafy mosses of Kuzedeevsky linden tree island at the mountain foot of Kuznetsk Alatau] Бот. Журн. [Bot.Zhurn.] 61(5): 712-718.

[GUDOSHNIKOV, S.V.] ГУДОШНИКОВ C.В. 1986. Флора листостебельных мхов черневого подпояса Южных гор Сибири и проблема происхождения черневой тайги. - [Moss flora of chernevoi belt of southern Mountains of Siberia and problem of the origin of chernevaya taiga] Томск, Изд. Томск. Ун-та [Tomsk, Izd. Tomsk Uni]. 192 pp.

[IGNATOV, M.S.] ИГНАТОВ М.С. 2012. Род Brachythecium Bruch, Schimp. \& Guembel. - Флора мхов России, http://arctoa.ru/Flora/taxonomy-ru/taxonomy-ru.php (версия 14.IV.2012).

IGNATOV, M.S., O.M. AFONINA, E.A. IGNATOVA et al. 2006. Checklist of mosses of East Europe and North Asia. - Arctoa 15: 1-130.

[IGNATOV, M.S. \& E.A. IGNATOVA] ИГНАТОВ M.C., Е.А. ИГНАТОВА 2003-2004. Флора мхов средней части Европейской России. T. 1-2. - [Moss flora of the Middle European Russia. Vols. 1-2] M., KMK [Moscow, KMK]: 1 (2003): 1-608; 2 (2004): 609-960.

IGNATOV, M.S. \& I.A. MILYUTINA 2010. The genus Brachythecium (Brachytheciaceae, Musci) in Russia: comments on species and key for identification. - Arctoa 19: 1-30.

[IGNATOVA, E.A.] ИГНАТОВА Е.А. 2012. Ключ для видов Schistidium [Key for Schistidium species]. - Флора мхов России [Moss flora of Russia], http://arctoa.ru/Flora/taxonomy-ru/taxonomy-ru.php (версия 8.II.2012).

IGNATOVA, E.A. \& V.E. FEDOSOV 2008. Dicranum with fragile leaves in Russia. - Arctoa 17: 63-83.

IGNATOVA, E.A. \& O.YU. PISARENKO 2013a. New moss records from Kemerovo Province. 3. - In: Sofronova E.V. (ed.) New bryophyte records. 2. Arctoa 22: 252-253.

IGNATOVA, E.A. \& O.YU. PISARENKO 2013b. New moss records from Novosibirsk Province. 3. - In: Sofronova E.V. (ed.) New bryophyte records. 2. Arctoa 22: 252

[ILINA, I.S., E.I. LAPSHINA, N.N. LAVRENKO et al.] ИЛЬИНА И.С., Е.И.ЛАПШИНА, Н.Н.ЛАВРЕНКО и др. 1985. Растительный покров Западно-Сибирской равнины. - [Plant cover of West-Siberian plain] Новосибирск, Наука [Novosibirsk, Nauka]. 251 pp.

[IOSCHENKO, E.N. \& N.N. LASHCHINSKIY] ИОЩЕНКО Е.Н., Н.Н. ЛАЩИНСКИЙ 1994. Пространственно-возрастная структура древостоя как функция биологии древесных пород. - [Spatial-age structure as a function of tree species biology] В кн.: Еленевский А.Г. (ред.) Успехи экологической морфологии растений и ее влияние на смежные науки. М., Прометей [In: Elenevsky, A.G. (ed.) Uspehi ekologicheskoy morfologii rasteny i ee vliyanie na smezhnye nauki. Moscow, Prometei]: 63-64.

[KASHMENSKAYA, O.V., O.V. KAZAKEVICH \& Z.N. SHVAREVA] КАШМЕНСКАЯ О.В., Ю.П. КАЗАКЕВИЧ, З.Н. ШВАРЕВА 1969. Кузнецкое нагорье. - [Kuznetskiy upland region] В кн.: Стрелков С.А., В.В. Вдовин (ред.) История развития рельефа Сибири и Дальнего Востока. Алтае-Саянская горная область. М., Наука [In: Strelkov, S.A. \& V.V.Vdovin (eds.) Istorija razvitija reliefa Sibiri $i$ Dalnego Vostoka. Altaie-Sajanskaja gornaja oblast. Moscow, Nauka]: 204-239.

[KHRAMOV, A.A. \& V.I. VALUTSKY] ХРАМОВ А.А., В.И. ВАЛУЦКИЙ 1977. Лесные и болотные фитоценозы Восточного Васюганья (Структура и биологическая продуктивность). - [Forest and swamp phytocoenosis of Eastern Vasyugan'e (Structure and biological productivity)] Новосибирск, Наука [Novosibirsk, Nauka]. 222 pp.

KOPONEN, T., E.A. IGNATOVA, O.I. KUZNETSOVA \& M.S. IGNATOV 2012. Philonotis (Bartramiaceae, Bryophyta) in Russia. - Arctoa 21: 21-62.

[KOROLYUK, A.YU.] КОРОЛЮК А.Ю. 1993а. Синтаксономия растительности юга Западной Сибири. 2. Луговая, степная и лесная растительность. - [Syntaxonomy of vegetation of south of West Siberia. 2. Meadow, steppe and forest vegetation] Новосибирск.
Рукопись; Депонир. В ВИНИТИ [Novosibirsk. Manuscript; deposited VINITI] 09.06.93. N 1578-B93. 49 pp.

[KOROLYUK, A.YU.] КОРОЛЮК А.Ю. 1993b. Структурная организация растительного покрова и методы ее изучения (на примере Барабинской равнины). - [Structural organization of plant cover and methods of its explonation (on example Baraba plain)] Дисс. . канд.биол. Наук, Новосибирск, ЦСБС СО РАН [Ph. D. Thesis, Novosibirsk, Centr. Sib. Bot. Sad.].

KOROLYUK, A. 1999. Phytosociological report from the saline habitats in SW Siberia and N Kazachstan. - In: Lieth, H. et al (eds.) Halophyte uses in different climates I.). Lieden, The Nitherlands: 133-146.

[KOROLYUK, A.YU.\& L.M. KIPRIYANOVA] КОРОЛЮК А.Ю., Л.М. КИПРИЯНОВА 2005. Растительные сообщества центральной Барабы (район озера Чаны). - [Plant Communities of the Central Baraba (the Lake Chany Region)] Сибирский экологический журнал [Sibirsky Ecologichesky Zhurn.] 12 (2): 193-200.

[KOROLYUK, A.YU., B.A. SMOLENTZEV, A.V. EGOROVA, I.A. KHRUSTALEVA \& O.M. MASLOVA] КОРОЛЮК А.Ю., Б.А. СМОЛЕНЦЕВ, А.В. ЕГОРОВА, И.А. ХРУСТАЛЕВА, О.М. МАСЛОВА 2007. К характеристике почвенно-растительного покрова луговостепных ложбин в ленточных борах юга Алтайского края. - [То the characteristic of the soil-vegetation cover of meadow-steppe hollows in steppe Pine forests in Altay Region] В кн.: Куприянов А.Н. (ред.) Ботанические исследования Сибири и Казахстана: сборник научных трудов. Кемерово, Ирбис [In: Kupriyanov, A.N. (ed.) Воtanicheskie issledovania Sibiri i Kazakhstana: sbornik nauchnih trudov. Kemerovo, Irbis] 17: 89-99.

[KOROLYUK, A.YU., B.A. SMOLENTZEV \& N.N. LASHCHINSKIY] КОРОЛЮК А.Ю., Б.А. СМОЛЕНЦЕВ, Н.Н. ЛАЩИНСКИЙ 2008. Почвенно-растительный покров приозерной равнины Кулундинского озера. - [Soil and vegetation cover of the circumlake plain of lake Kulunda] Растительный мир Азиатской России [Rastitel 'nyi mir Asiatskoj Rossii] 2: 80-88.

[KRYLOV, P.N.] КРЫЛОВ П.Н. 1891. Липа на предгорьях Кузнецкого Алатау. - [Linden in the foothills of Kuznetskiy Alatau] Изв. Импер. Томск. Ун-ma [Izv. Imper. Tomsk Uni]: 1-40.

[KRYLOV, P.N.] КРЫЛОВ П.Н. 1898. Очерк растительности Томской губернии. - [Essay on vegetation of Tomsk Province] Томск [Tomsk]. $26 \mathrm{pp}$.

[KRYLOV, P.N.] КРЫЛОВ П.Н. 1919. Очерк растительности Сибири. - [Essay on vegetation of Siberia] Статистико-экономический бюллетень, Томск [Statistiko-ekonomichesky byulleten, Tomsk] 17: $1-24$.

[KRYLOV, P.N.] КРЫЛОВ П.Н. 1924. Материалы к флоре споровых растений Алтая и Томской губернии.. - [Contributions to the flora of spore plants of Altai and Tomsk Province] Изв. Томск. Гос. Ун-та [Izv. Tomsk Gos. Uni] 75: 1-48.

[KUMINOVA, A.V.] КУМИНОВА А.В. 1950. Растительность Кемеровской области. - [Vegetation of Kemerovo Province] Новосибирск [Novosibirsk], 167 pp.

[KUMINOVA, A.V., T.A.VAGINA \& E.D. LAPSHINA] КУМИНОВА А.В., Т.А. ВАГИНА, Е.Д. ЛАПШИНА 1963. Геоботаническое районирование юго-востока Западно-Сибирской низменности. [Geobotanical Regional Assignment of the South-Eastern Part of the West-Siberian Lowlands] В кн.: Куминова А.В. (ед.) Тр. ЦСБС., Bылn. 6. Растительность степной и лесостепной зон Западной Сибири. Новосибирск, СО РАН CCCP [In: Kuminova A.V. (ed.) Trudi CSBS, vip.6. Rastitelnost stepnoi I lesostepnoi zon Zapadnoi Sibiri Vegetation of the steppe and wood steppe zones of Western Siberia. Novosibirsk, SB AS USSR]: 35-62.

[LAPSHINA, E.D.] ЛАПШИНА Е.Д. 2003. Флора болот юго-востока Западной Сибири. - [Flora of mires of southeastern part of West Siberia] Томск [Tomsk], $296 \mathrm{pp}$.

[LAPSHINA, E.D., A.YU. KOROLYUK, V. BLOITEN, E.YA. MULDIYAROV \& V.I. VALUTSKY] ЛАПШИНА Е.Д., А.Ю. КОРОЛЮК, В.БЛОЙТЕН, Е.Я. МУЛЬДИЯРОВ, В.И. ВАЛУЦКИЙ 2000 Структура растительного покрова западной части Большого 
Васюганского болота (на примере ключевого участка «Узас»). [Structure of Vegetation Cover of the Western Part of the Great Vasyugan Swamp (on the Example of the Key Site "Uzas"] Сиб. Экол. Журн. [Sibirsky Ecologichesky Zhurn.] 7(5): 563-576.

[LAPSHINA, E.D \& E.YA. MULDIYAROV] ЛАПШИНА Е.Д., Е.Я. МУЛЬДИЯРОВ 1995. К характеристике болот заповедника «Кузнецкий Алатау». - [Tо characterization of bogs of Kuznetskiy Alatau Reserve] В кн.: Васильченко А.А., Н.Н. Лащчинский (ред.) Биоценотические исследования в заповеднике «Кузнеикий Алатау». Bылn. 1. Новосибирск. [In: Vasilchenko A.A., N.N. Lashchinsky (eds.) Biotsenoticheskie issledovaniya v zapovednike "Kuznetskiy Alatau”. 3. Novosibirsk] 1: 47-58.

LAPSHINA, E.D. \& E.YA. MULDIYAROV 1998. The bryophyte flora of the Middle Western Siberia. - Arctoa 7: 25-32.

[LASHCHINSKY, N.N.] ЛАЩИНСКИЙ Н.H. 1996. Ценофлора высокотравных лугов заповедника «Кузнецкий Алатау». - [Coenoflora tall-herbaceous meadows of Kuznetskiy Alatau Reserve] $В$ кн.: Васильченко А.А., Н.Н. Лашинский (ред.) Биоценотические исследования в заповеднике "Кузнечкий Алатау». Новосибирск [In: Vasilchenko, A.A., N.N. Lashchinsky (eds.) Biotsenoticheskie issledovaniya v zapovednike "Kuznetskiy Alatau”. Novosibirsk] 2: 53-60.

[LASHCHINSKIY, N.N.] ЛАЩИНСКИЙ Н.Н. 2009. Растительность Салаирского кряжа - [Vegetation of Salair ridge] Новосибирск, Изд. Гeo [Novosibirsk, Izd. Geo]. 263 pp.

[LASHCHINSKY, N.N. \& N.V. DEMIDENKO] ЛАЩИНСКИЙ Н.Н., Н.В. ДЕМИДЕНКО 2005. Некоторые характеристики снежного покрова в лесном поясе Кузнецкого Алатау. - [Some characteristics of the snow cover in the forest belt of Kuznetskiy Alatau] $В \kappa н$.: Еремеева Н.И., Д.В. Сущев (ред.) Труды Кемеровского отделения Русского энтомологического общества. Кемерово [In: Yеrетееva, N.I. \& D.V. Sushchev (eds.) Trudi Kemerovskogo otdelenija Russkogo entomologicheskogo obschestva. Kemerovo] 3: 41-47.

[LASHCHINSKIY, N.N. \& A.YU. KOROLYUK] ЛАЩИНСКИЙ Н.Н., А.Ю. КОРОЛЮК 2012. Синтаксономия высших единиц лесной растительности южной тайги Западно-Сибирской равнины и гумидных низкогорий Алтае-Саянской горной области. - [High syntaxonomical units of forest vegetation from southern taiga of West Siberian Plain and from humid low mountains of Altai-Sayan mountain area] Изв. Самаровского Научного Центра РАН [Izv. Samarovskogo Nauchnogo Centra RAN] 14, 1(4): 1047-1049.

[LASHCHINSKY, N.N. \& N.V. LASHCHINSKAYA] ЛАЩИНСКИЙ Н.Н., Н.В. ЛАЩИНСКАЯ 2012. Травяные мелколиственные леса овражно-балочных систем Приобского плато (Западная Сибирь). - [Small-leaved herbaceous forests in galley and ravine systems of Priobskoe Plateau] Paстительность Poсcиu. [Vegetation of Russia. St. Peterburg] 21: 78-96.

[LASHCHINSKY, N.N., N.I. MAKUNINA \& A.F. GULYAEVA] ЛАЩИНСКИЙ Н.Н., Н.И. МАКУНИНА, А.Ф. ГУЛЯЕВА 2011. Структура растительного покрова древних террас реки Томь в центральной части Кузнецкой котловины. - [Spatial structure of vegetation cover on ancient Tom' River Terraces in central part of Kuznetskaya depression] Растительный мир азиатской России [Rastitel 'nyi mir Asiatskoj Rossii] 1(7): 55-65.

[LASHCHINSKY, N.N., N.I. MAKUNINA, O.YU. PISARENKO \& A.F. GULYAEVA] ЛАЩИНСКИЙ Н.Н., Н.И. МАКУНИНА, О.Ю. ПИСАРЕНКО, А.Ф. ГУЛЯЕВА 2011. Ландшафтообразующая растительность северной части Мелафировой подковы (Кемеровская область). - [Landscape-forming vegetation of Melafir Horseshoe northern part (Kemerovo oblast) Растительный мир азиатской Poccuu [Rastitel'nyi mir Asiatskoj Rossii] 2(8): 85-99.

[LASHCHINSKY, N.N. \& O.YU. PISARENKO] ЛАЩИНСКИЙ Н. Н., О.Ю. ПИСАРЕНКО 1999. Бриофлора степей Салаирского кряжа. - [Bryoflora of Salair steppe] Бот. Журн. [Bot. Zhurn.] 84(4): 79-85.

[LASHCHINSKY, N.N. \& O. YU. PISARENKO] ЛАЩИНСКИЙ Н.Н., О.Ю. ПИСАРЕНКО 2010. Ленточные болота междуречья рек Кия и Яя (Западная Сибирь). - [Ribbon mires in Kija-Yaya interfluve (Western Siberia)] Растительный мир азиатской России [Rastitel 'nyi mir Asiatskoj Rossii] 1(5): 42-48.
[LASHCHINSKIY, N.N., M.P. TISHCHENKO, O.YU. PISARENKO \& N.V. LASHCHINSKAYA] ЛАЩИНСКИЙ Н.Н., М.П. ТИЩЕНКО, О.Ю. ПИСАРЕНКО, Н.В. ЛАЩИНСКАЯ 2014. РаститеЛЬНЫЙ пОкров подтаежных ландшафтов предгорной равнины правобережья Оби. - [Vegetation cover of subtaiga landscapes in peneplain on a right bank of Ob river] Растительность России. [Vegetation of Russia. St. Peterburg] in press.

[LAVRENKO, Е.M.] ЛАВРЕНКО Е.M. 1978. О растительности степей и пустынь Монгольской Народной Республики. - [On the vegetation of steppe and deserts of Mongolian People's Republic] Проблемы освоения пустынь [Problemy osvoeniya pustyn] 1: 3-19.

[LAVRENKO, Е.M., Z.V.KARAMYSHEVA \& L.I. NIKULINA] ЛAВPEHКО Е.М., З.В. КАРАМЫШЕВА, Л.И. НИКУЛИНА 1991. Степи Евразии. - [Steppes of Eurasia] Л., Наука [Leningrad, Nauka]. 145 pp.

[LISS, O.L. \& N.A. BEREZINA] ЛИСС О.Л., Н.А. БЕРЕЗИНА 1981. Болота Западно-Сибирской равнины. - [Mires of West Siberian Plain] М., Изд. Мос. ун-та [Moscow, Izd Mosc. Uni]. 204 pp.

[LOGUTENKO, N.V.] ЛОГУТЕНКО Н.В. 1963. Материалы к изучению бриофлоры лугов и болот лесостепной и степной зон Западной Сибири. - [Materials to the study of the meadows and mires bryoflora of foreststeppe and steppe zones of Western Siberia] $B$ кн.: Куминова А.В. (ед.) Тр. ЦСБС., вып. 6. Растительность степной и лесостепной зон Западной Сибири. Новосибирск, Изд. СО АН CCCP [In: Kuminova, A.V. (ed.) Trudi CSBS, vip.6. Rastitelnost stepnoi I lesostepnoi zon Zapadnoi Sibiri Vegetation of the steppe and wood steppe zones of Western Siberia. Novosibirsk, Izd. SB AS USSR]: 334-337.

[MAKUNINA, N.I.] МАКУНИНА Н.И. 1998. Биоразнообразие и структура растительности межгорных котловин северной части Алтае-Саянской горной области. - [Biodiversity and structure of vegetation of intermountain depressions in northern part of the AltaiSayan region] Дисс. ... к-та биол. Наук, Новосибирск, ЦСБС СО PAH [Ph. D. Thesis, Novosibirsk, Centr. Sib. Bot. Sad.].

[MAKUNINA, N.I. \& T.V. MALTSEVA] МАКУНИНА Н.И., Т.В. МАЛЬЦЕВА 2008. Растительность лесостепных и подтаежных предгорий Алтае-Саянской горной области. - [The Vegetation of Forest-steppe and Subtaiga foothills of Altai-Sayani Mountain System] Сиб. Бот. Вестн.: электрон. журн. [Sibirsky botanichesky vestnik: electronic journal] 3(1-2): 45-156. http://journal.csbg.ru/ ?User_current_article_id $=54 \&$ user_action $=$ show_article

[MAKUNINA, N.I., KOROLYUK A.YU. \& T.V. MALTSEVA] MAKYНИНА Н.И., А.Ю. КОРОЛЮК, Т.В.МАЛЬЦЕВА 2010. РаститеЛЬность Бийско-Чумышской возвышенности. - [The vegetation of the Bya-Chumysh upland] Растительность России. [Vegetation of Russia. St. Peterburg] 16: 40-55.

[MULDIYAROV, E.YA. \& N.A. CHERNOVA] МУЛЬДИЯРОВ Е.Я., Н.А. ЧЕРНОВА 2002. Новые виды мохообразных Томской области [New records of bryophytes in Tomsk Province]-Arctoa 11: 215-218.

[MULDIYAROV, E.YA. \& E.D. LAPSHINA] МУЛЬДИЯРОВ Е.Я., Е.Д. ЛАПШИНА 1996. Роль мохообразных в растительных сообществах болот заповедника “Кузнецкий Алатау” - [Role of bryophytes in bog plant communities of Kuznetskiy Alatau Reserve Reserve (South of West Siberia)] В кн.: Васильченко А.А., Н.Н. Лашинский (ред.) Биоиенотические исследования в заповеднике "Кузнеикий Алатау". Новосибирск [In: Vasilchenko, A.A. \& N.N. Lashchinsky (eds.) Biotsenoticheskie issledovaniya v zapovednike "Kuznetskiy Alatau". Novosibirsk] 2: 67-77.

[MULDIYAROV, E.YA. \& E.D. LAPSHINA] МУЛЬДИЯРOB Е.Я., Е.Д. ЛАПШИНА 1998. Новые для лесной зоны Западной Сибири виды мохообразных. - [Bryophytes new for forest zone of Western Siberia] Бот. Журн. [Bot. Zhurn.] 83 (10): 124-127.

[MULDIYAROV, E.YA. \& E.D. LAPSHINA] МУЛЬДИЯРOB Е.Я., Е.Д. ЛАПШИНА 2005. К изучению болот Кузнецкого Алатау - [Study of the Kuznetsk Alatau Swamps] Сиб. Экол. Журн. [Sibirsky Ecologichesky Zhurn.] 7(5): 645-652.

[MULDIYAROV, E.YA., E.D. LAPSHINA \& A.L. BORISENKO] МУЛЬДИЯРОВ Е.Я., Е.Д. ЛАПШИНА, А.Л. БОРИСЕНКО 2013. 
Мохообразные. - [Bryophyta] B кн: Адам А.М. (ред.) Красная книга Томской области. Издание 2-е. Томск, "Печатная мануфактура" [In: Adam, A.M. (ed.) Red data book of Tomskaya province $2 d$ ed. Tomsk, Pechatnaya Manufactura]: 403-425.

[MULDIYAROV, E.YA., A.I. PYAK \& A.L. EBEL] МУЛЬДИЯРОВ Е.Я., А.И. ПЯК, А.Л. ЭБЕЛЬ 1996. Новые для флоры Томской области виды мохообразных и сосудистых растений. - [The new species of mosses and vascular plants for flora of the Tomsk region] Бom. Журн. [Bot. Zhurn.] 81(5): 90-93.

[MYACHKOVA, N.A.] МЯЧКОВА Н.А. 1983. Климат СCCP. - [Climate of USSR] М., Изд. Моск. Универс. [Moscow, Izd. Mosc. Uni]. $192 \mathrm{pp}$.

[NIKOLAEV, V.A.] НИКОЛАEB В.A. 1970. Геоморфологическое районирование Западно-Сибирской равнины. - [Geomorphological zonation of West-Siberian plain] В кн.: Стрелков С.А., В.В. Вдовин (ред.) История развития рельефа Сибири и Дальнего Востока. Алтае-Саянская горная область. M., Наука [In: Strelkov, S.A. \& V.V. Vdovin (eds.) Istorija razvitija reliefa Sibiri i Dalnego Vostoka. Altaie-Sajanskaja gornaja oblast. Moscow, Nauka]: 226-254.

[NIKOLAEV, V.A.] НИКОЛАЕВ В.А. 1972. Геолого-геоморфологический очерк. - [Geologo-geomorphological study] В кн.: Полубаринова-Кочина П.Я. (ред.) Кулундинская степь и вопросы ее мелиорации. Новосибирск, Наука [In: Polubarinova-Kochina, P.Ya. (ed.) Kulunda steppe and questions of its melioration. Novosibirsk, Nauka]: 194-204.

[NIKOLAEV, V.A.] НИКОЛАЕВ В.А. 1988а. Геоморфологическое районирование. - [Geomorphological zoning ] В кн.: Кашменская, О.В. (ред.) Рельеф Западно-Сибирской равнины. Новосибирск, Наука [In: Kashmenskaya O.V. (ed.) Relief of West-Siberian plain. Novosibirsk, Nauka]: 125-130.

[NIKOLAEV, V.A.] НИКОЛАЕВ В.А. 1988b. Геоморфологическое районирование. - [Geomorphological zoning] В кн.: Кашменская, O.В. (ред.) Рельеф Западно-Сибирской равнины. Новосибирск, Наука [In: Kashmenskaya, O.V. (ed.) Relief Zapadno-Sibirskoi ravnini. Novosibirsk, Nauka]: 139-145.

[NIKOLAEV, V.A.] НИКОЛАЕВ В.А. 1988c. Геоморфологическое районирование сельско-хозяйственной зоны Западно-Сибирской равнины. - [Geomorphological zoning of agricultural zone of WestSiberian plain] В кн.: Кашменская О.В. (ред.) Рельеф ЗападноСибирской равнины. Новосибирск, Наука [In: Kashmenskaya, O.V. (ed.) Relief Zapadno-Sibirskoi ravnini. Novosibirsk, Nauka]: 139145.

[NIKOLAEV, V.A., B.V. MIZEROV, N.P. BELETSKAYA, S.G. VODOPIYANOV, V.I. GORBUNOV, A.G. GRITSENKO, I.V. PILKEVICH, D.V. PUCHKOVA, D.S. SINEL'NIKOV \& A.S. SHEVNIN] НИКОЛАЕВ В.А., Б.В. МИЗЕРОВ, Н.П. БЕЛЕЦКАЯ, С.Г. ВОДОПЬЯНОВА, В.И. ГОРБУНОВ, А.Г. ГРИЦЕНКО, И.В. ПИЛЬКЕВИЧ, Д.В. ПУЧКОВА, Д.С. СИНЕЛЬНИКОВ, А.С. ШЕВНИН 1982. Рельеф южных равнин Западной Сибири. - [Relief of south plains of West-Siberia] В кн.: Николаев В.A. (ред.) Закономерности развития рельефа Северной Азии, Новосибирск, Наука [In: Nikolaev, V.A. (ed.) Zakonomernosti razvitiya reliefa Severnoi Azii, Novosibirsk, Nauka]: 11-47.

[NOZHINKOV, A.E.] НОЖИНКОВ А.Е. 2004. Листостебельные мхи ленточных сосновых боров Алтайского Края. - [Leafy mosses of pine forest of Altai Region] В кн.: Куприянов А.Н. (ред.) Ботанические исследования Сибири и Казахстана: сборник научных трудов. Барнаул, Изд. Алтайского Гос. Унив-та [In: Kupriyanov, A.N. (ed.) Botanicheskie issledovania Sibiri i Kazakhstana: sbornik nauchnih trudov. Barnaul, Izd. Altai Gos. Uni] 10: 90-96.

[NOZHINKOV, A.E.] НОЖИНКОВ А.Е. 2006. Список листостебельных мхов Алтайского края. - [List of mosses of of Altai Region] $B$ кн.: Куприянов А.Н. (ред.) Ботанические исследования Сибири и Казахстана: сборник научных трудов. Барнаул-Кемерово, Ирбис [In: Kupriyanov, A.N. (ed.) Botanicheskie issledovania Sibiri i Kazakhstana: sbornik nauchnih trudov. Barnaul-Kemerovo, Irbis] 12: 56-67.

[NOZINKOV, A.E. \& O.YU. PISARENKO] НОЖИНКОВ A.Е., O.Ю.
ПИСАРЕНКО 2008. К бриофлоре Горной Шории. - [On the bryoflora of Mountain Shoria] В кн.: Куприянов A.Н. (ред.) Ботанические исследования Сибири и Казахстана: сборник научных трудов. Кемерово, Ирбис [In: Kupriyanov, A.N. (ed.) Botanicheskie issledovania Sibiri i Kazakhstana: sbornik nauchnih trudov. Kemerovo, Irbis] 14: 24-29.

[NOZHINKOV, A.E. \& G.S. TARAN] НОЖИНКОВ A.Е., Г.C. ТАРAH 2008. К бриофлоре поймы Оби в Быстроистокском районе Алтайского края. - [On bryoflora of Ob River floodplain in Bytstry Istok Distr. Of Altaysky Territory] Вестник ТГУ. Биология [Vestnik Tomsk. Gos. Uni, Ser. Biol.] 3(4): 35-41.

[OLYUNIN, V.N.] ОЛЮНИН В.Н. 1975. Горы Южной Сибири. Геоморфологическое районирование. - [Mountains of South Siberia. Geomorphological zoning] В кн.: Коржуев С.С. (ред.) Равнины и горы Сибири. М.: Наука [In: Korzhuev, S.S. (ed.) Plains and mountains of Siberia. Moskow, Nauka]: 245-328.

[PILNIKOVA, Z.N. (ed.)] ПИЛЬНИКОВА 3.Н. (ред.) 1993. Научноприкладной Справочник по климату СССР. Серия 3. Многолетние данные. Томская, Новосибирская, Кемеровская области, Алтайский край. - [Scientific and Applied Climate Reference Book of the USSR. Series 3. Long-term data. Tomsk, Novosibirsk, Kemerovo Provinces and Altai Territory] СПб, Гидрометеоизат [St-Petersburg, Gidrometeoizdat] 20(1-6): 717 pp.

PISARENKO, O.Yu. 1999. Bryophyte communities of Salair forests (South Siberia). -Arctoa 8: 35-49.

PISARENKO, O.Yu. 2004. Mosses of the central part of Kuznetskiy Alatau (Southern Siberia). - Arctoa 13: 241-260.

PISARENKO, O.Yu. 2006. On the variability and ecology of Pterygoneurum subsessile and P. Kozlovii (Pottiaceae, Bryophyta). - Arctoa 15: $169-182$.

PISARENKO, O.Yu. 2007a. New moss records from Kemerovo Province 1. - Arctoa 16: 191-192.

[PISARENKO, O.YU.] ПИСАРЕНКО О.Ю. 2007b. Листостебельные мхи. - [Mosses] В кн.: Лашинский Н.Н. (ред.) Флора Салаирского кряжа. Новосибирск, Гео [In: Lashchinsky, N.N. (ed.) Flora Salairskogo kriaja. Novosibirsk, Geo]: 137-154.

[PISARENKO, O.YU.] ПИСАРЕНКО О.Ю. 2008. Моховидные. [Bryophytes] В кн.: Марченко Ю.Ю. (ред.) Красная Книга Новосибирской области: животные, растения и грибы. Новосибирск, Apma [In: Marchenko, Yu.Yu. (ed.) Red Data Book of Novosibirsk Province: animals, plants and fungi. Novosibirsk, Arta]: 443-465.

[PISARENKO, O.YU.] ПИСАРЕНКО О.Ю. 2009а. Материалы по бриофлоре заповедника «Кузнецкий Алатау». - [On the bryoflora of Kuznetskiy Alatau nature reserve] В кн.: Бабина С.Г. (ред.) Биологическое разнообразие. - определяющие факторы, мониторинг: матер. Научной конф., посвященной 20-летию заповедника (Междуреченск, 16-18 сентября 2009 г). Кемерово, “Азия” [In: Babina, S.G. (ed.) Biologicheskoye raznoobraziye - opredeliayuschie faktori, monitoring: materialy naychnoj konferentsii, posviaschennoj 20-letiyu zapovednika (Mezdurechensk, 16-18 September 2009). Kemerovo, "Asia"]: 147-148.

[PISARENKO, O.YU.] ПИСАРЕНКО О.Ю. 2009b. Материалы по новым и редким видам мхов Алтае-Саянского экорегиона. - [On the new and rare moss species of Altai-Sayan ecoregion] $В$ кн.: Шмаков А.И. (ред.) Проблемы ботаники Южной Сибири и Монголии: материалы VIII междунар. Научно-практической конф. (Барнаул, 19-22 октября 2009 г). Барнаул, РПК “Аrtика” [In: Shmakov, A.I. (ed.) Problemy botaniki juzhnoi Sibiri i Mongolii: materialy VIII mezdunarodnoi konferentsii (Barnaul, 19-22 October 2009). Barnaul, RPK “Artika”]: 89-91.

[PISARENKO, O.YU.] ПИСАРЕНКО О.Ю. 2011. Листостебельные мхи. - [Mosses] В кн.: Куприянов А.Н. (ред.) Растительный мир Караканского хребта. Новосибирск, Гео [In: Kupriyanov, A.N. (ed.) Rastitel 'nii mir Karakanskogo khrebta. Novosibirsk, Geo]: 84-88.

PISARENKO, O.Yu. 2012a. New moss records from Altaisky Territory. 2. - In: Sofronova, E.V. (ed.) New bryophyte records. 1. Arctoa 21: 290. 
PISARENKO, O.Yu. 2012b. New moss records from Kemerovo Province. 2. - In: Sofronova, E.V. (ed.) New bryophyte records. 1. Arctoa 21: 290 .

PISARENKO, O.Yu. 2012c. New moss records from Novosibirsk Province. 2. - In: Sofronova, E.V. (ed.) New bryophyte records. 1. Arctoa 21: 289 .

[PISARENKO, O.YU.] ПИСАРЕНКО О.Ю. 2013. Мхи центральной части Колывань-Томской дуги и их представленность на территории ЦСБС СО РАН. - [Mosses of central part of Kolivan-Tomsk region and their representation in the territory of CSBG SB RAS] $B \kappa \mu$.: Красников А.А. (ред.) Растительный мир Северной Азии: проблемы изучения и сохранения биоразнообразия. Материаль Всероссийской конференции (Новосибирск, 1-3 октября 2013). [In: Krasnikov, A.A. (ed.) Rastitel'nyi mir Severnoj Asii. Materialy Vserossiyskoi konferentsii (Novosibirsk, 1-3 October 2013] Новосибирск, ЦСБC CO PAH [Novosibirsk, CSBG SB RAS]: 109-111.

[PISARENKO, O.YU. \& N.N. LASHCHINSKY] ПИСAРЕНКО O.Ю., Н.Н. ЛАЩИНСКИЙ 1999. Бриофлористическая характеристика растительных сообществ Салаирского кряжа. - [Bryofloristic characteristic of Salair vegetative communities] В кн.: Куприянов А.Н. (ред.) Ботанические исследования Сибири и Казахстана: сборник научных трудов. Барнаул, Изд. Алтайского Гос. Унив-та [In: Kupriyanov, A.N. (ed.) Botanicheskie issledovania Sibiri i Kazakhstana: sbornik nauchnih trudov. Barnaul, Izd. Altai Gos. Uni] 5: 58-67.

PISARENKO, O.YU., E.A. IGNATOVA \& M.S. IGNATOV 2001. Entostodon hungaricus (Boros) Loeske (Funariaceae, Musci) in Altaisky territory, South Siberia. - Arctoa 10: 97-102.

[PISARENKO, O.YU., E.D. LAPSHINA \& E.YA. MULDIYAROV] ПИСАРЕНКО О.Ю., Е.Д. ЛАПШИНА, Е.Я. МУЛЬДИЯРОВ 2011. Ценотические позиции и экологические амплитуды мхов в растительных сообществах Большого Васюганского болота. [Cenotic Positions and Ecological Amplitudes of Mosses in the Vegetation of the Great Vasyugan Swamp] Сиб. Экол. Журн. [Sibirsky Ecologichesky Zhurn.] 3: 379-393 .

PISARENKO, O.YU., A.E. NOZINKOV \& D.V. ZOLOTOV 2007. New moss records from Altaisky Territory 1. - Arctoa 16: 190-191.

[PISARENKO, O.YU. \& A.E. NOZINKOV] ПИСAРЕНКО O.Ю., A.Е. НОЖИНКОВ 2007. Материалы по редким видам мхов Кемеровской области. - [On the rare moss species of Kemerovo Province] $B$ кн.: Куприянов А.Н. (ред.) Флора и растительность антропогенно нарушенных территорий. Кемерово, Ирбис [In: Kирriуanov, A.N. (ed.) Flora i rastitelnost antropogenno narushennih territorj. Kemerovo, Irbis] 3: 49-56.

[PISARENKO, O.YU. \& A.E. NOZINKOV] ПИСAРЕНКО O.Ю., А.Е. НОЖИНКОВ 2012. Мохообразные. - [Bryophytes] В кн.: Куприянов А.Н. (ред.) Красная Книга Кемеровской области: редкие и находящиеся под угрозой исчезновения виды растений и грибов. T.1. Кемерово: “Азия принт” [In: Kuprijanov, A. N. (ed.) Red Data Book of Kemerovo Provincei. Kemerovo, Asia print]: 156-165.

[PISARENKO, O.YU., A.E. NOZINKOV \& D.V. ZOLOTOV] ПИCAРЕНКО О.Ю., А.Е. НОЖИНКОВ, Д.В. ЗОЛОТОВ 2008. К бриофлоре озерно-болотных комплексов Алтайского края. - [On the bryoflora of lake-mire complexes of Altai Terrytories] В кн.: Шмаков А.И. (ред.) Проблемы ботаники Южной Сибири и Монголии. Материалы 7-й международной научно-практической конференции, Барнаул, 22-24 октября 2008 г.. Барнаул, РПК "Artика" [In: Shmakov, A.I. (ed.) Problemi botaniki juznoi Sibiri $i$ Mongolii. Materiali 7 mezdunarodnoi konferencii, Barnaul, 22-24 October 2008. Barnaul, RPK “Artika”]: 260-263.

[PISARENKO, O.YU. \& V.I. VALUTSKIY] ПИСАРЕНКО О.Ю., В.И. ВАЛУЦКИЙ 2013. Мхи Академгородка. - [Mosses of Academgorodok] В кн.: Жимулев И.Ф. (ред.) Динамика экосистем Новосибирского академгородка. Новосибирск, Изд. СО РАH [In: Zhimиlev, I.F. (ed.) Dynamica ecosystem Novosibirskogo Akademgorodka. Novosibirsk, Izd. SB RAS]: 148-153.

[POLIKARPOV, N.P., N.M. TCHEBAKOVA \& D.I. NAZIMOVA] ПОЛИКАРПОВ Н.П., Н.М. ЧЕБАКОВА, Д.И. НАЗИМОВА 1986. Климат и горные леса Южной Сибири. - [Climate and mountain forests of South Siberia] Новосибирск, Наука [Novosibirsk, Nauka]. $226 \mathrm{pp}$.

[POLOZHII, A.V. \& E.D. KRAPIVKINA] ПОЛОЖИЙ А.В., Э.Д. КРАПИВКИНА 1985. Реликты третичных широколиственных лесов во флоре Сибири. - [Tertiary relic of broadleaf deciduous forests in Siberian flora]. Томск, Изд. Томск. Ун-та [Tomsk, Izd. Tomsk Uni]. $158 \mathrm{pp}$.

[SEDELNIKOV, V.P.] СЕДЕЛЬНИКОВ В.П. 1979. Флора и растительность высокогорий Кузнецкого Алатау. - [The flora and vegetation of Kuznetskiy Alatau high-mountains] Новосибирск, Наука [Novosibirsk, Nauka]. $168 \mathrm{pp}$.

[SHPIN`, P.S.] ШПИНЬ П.С. 1980. Оледенение Кузнецкого Алатау. [Glaciation of Kuznetskiy Alatau] M., Наука [Moscow, Nauka]. 84 pp.

[VAGINA, T.А.] ВАГИНА Т.А. 1962. Луга Барабы. - [Baraba meadows] Новосибирск, Изд. СО АН СCCP [Novosibirsk, Izd. SB AS USSR]. $198 \mathrm{pp}$.

[VAGINA, T.A.] ВАГИНА Т.А. 1963. Засоленные луга Барабы и Кулунды. - [Saline meadows of Baraba and Kulunda] В кн.: Куминова А.В. (ед.) Тр. ЦСБС., Вып. 6. Растительность степной и лесостепной зон Западной Сибири. Новосибирск, Изд. СО АН СССР [In: Kuminova, A.V. (ed.) Trudi CSBS, vip.6. Rastitelnost stepnoi $i$ lesostepnoi zon Zapadnoi Sibiri Vegetation of the steppe and wood steppe zones of Western Siberia. Novosibirsk, Izd. SB AS USSR]: 163182.

[VALUTSKIJ, V.I.] ВАЛУЦКИЙ В.И. 2011 Растительность лесостепных рямов в восточной Барабе [Vegetation of forest-steppe riams in the eastern part of Baraba]. - Turczaninowia, 14(1): 109-119.

[VANDAKUROVA, E.V.] ВАНДАКУРОВА Е.В. 1950. Растительность Кулундинской степи. - [Vegetation of Kulunda steppe] Новосибирск, Изд. СО AH CCCP [Novosibirsk, Izd. SB AS USSR]. 128 pp.

[VANDAKUROVA, E.V.] ВАНДАКУРОВА Е.В. 1957. Ботаникогеографическое районирование Новосибирской области. - [Botanical-geographic zoning of Novosobirsk Province] В кн.: Абрамович Д.И. (ред.) Изв. Новосибирского отд. Географического общества СССР. Новосибирск, Изд. СО АН СССР [In: Abramovich, D.I. (ed.) Izv. Novosibirskogo otd. Geoeraficheskogo obschestva USSR. Novosibirsk, Izd. SB AS USSR] 1: 3-14.

[VASILJEV, A.N.] ВАСИЛЬЕВ А.Н. 1973. Редкие виды во флоре мхов Кузнецкого Алатау. - [Rare species in Kuznetsk Alatau bryoflora] Изв. CO AH CCCP, сер. биол. . [Izv. SB SA USSR, ser. Biol] 1(5): 126-128.

[VASILJEV, A.N.] ВАСИЛЬЕВ А.Н. 1974. Материалы по флоре листостебельных мхов Кузнецкого Алатау. - [On the moss flora of the Kuznetskiy Alatau] Вопросы ботаники и физиологии растений. Красноярск [Voprosy botaniki i fisiologii rastenij] 5: 35-47.

[VASILJEV, A.N.] ВАСИЛЬЕВ А.Н. 1975. К флоре мхов «липового острова» на Кузнецком Алатау. - [On moss flora of „linden“island of Kuznetsk Alatau] Изв. СО АН СCCP, сер. биол. [Izv SB SA USSR. ser. biol.] 5(1): 16-20.

[VASILJEV, A.N.] ВАСИЛЬЕВ А.Н. 1976. Листостебельные мхи избыточно увлажненных местообитаний Кузнецкого Алатау. [Mosses of surplus moist habitats of Kuznetskiy Alatau] В кн.: Глебов Ф.3. (ред.) Теория и практика лесного болотоведения и гидролесомелиораиии. Красноярск, Ин-т леса и древесины СО АН CCCP [In: Glebov, F.Z. (ed.) Teoriya i praktika lesnogo bolotovedeniya i gidrolesomelioratsii. Krasnoyarsk, Ins-t lesa I drevesini SB AS USSR]: 98-105.

[VASILJEV, A.N.] ВАСИЛЬЕВ А.Н. 1978. Листостебельные мхи Кузнецкого Алатау. - [Mosses of Kuznetskiy Alatau] Дисс. ... Канд.биол. Наук, Свердловск [Ph. D. Thesis, Sverdlovsk].

[VASILJEV, A.N.] ВАСИЛЬЕВ А.Н. 1995. Бриофлора центральной части Южной Сибири. - [Bryoflora of central part of South Siberia] Дисс. .. Д-ра. Биол. Наук, Новосибирск, ЦСБС СО РАН [Dr. Sci. Thesis, Novosibirsk, Centr. Sib. Bot. Sad.].

[VDOVIN, V.V.] ВДОВИН В.В. 1988. Кузнецко-Салаирская провинция. - [Kuznetsk-Salair Province] В кн.: Николаев В.А., Г.А. Чернов (ред.) Рельеф Алтае-Саянской горной области. Новосибирск, 
Наука [In: Nikolaev, V.A.\& G.A. Chernov (eds.) Relief Altaie-Sajanskoi gornoi oblasti. Novosibirsk, Nauka]: 40-71.

[VOLKOVA, I.I. \& E.YA. MULDIYAROV] ВОЛКОВА И.И., Е.Я. МУЛЬДИЯРОВ 2000. Дополнение к списку видов мохообразных болот заповедника “Кузнецкий Алатау”. - [Supplement to bryophytes list of bogs from Reserve (South of West Siberia)] В кн.: Васильченко А.А., Н.Н. Лащинский (ред.) Биоченотические исследования в заповеднике "Кузнеикий Алатау”. Кемерово [In: Vasilchenko, A.A. \& N.N. Lashchinsky (eds.) Biotsenoticheskie issledovaniya vapovednike Kuznetskiy Alatau. Kemerovo] 3: 36-40.

[ZARUTSKAYA, I.P. (ed.)] ЗАРУЦКАЯ И.П. (ред.) 1978. Алтайский край. Атлас. - [Altaisky territory. Atlas.] М.-Барнаул, ГУГК [Moscow-Barnaul, Izd. Glavnogo upravlenia geodezii i kartografii] (X), $222 \mathrm{pp}$.

ZOLOTOV, V.I. 2000. The genus Bryum (Bryaceae, Musci) in the Middle European Russia. - Arctoa 9: 155-232.

[ZOLOTOV, V.I.] ЗОЛОТОВ В.И. 2011. Bryum Hedw

. - Флора мхов России России [Moss flora of Russia] http://arctoa.ru/ Flora/taxonomy-ru/taxonomy-ru.php (версия 1.V.2011)

[ZYAT'KOVA, L.K.] ЗЯТЬКОВА Л.К. 1977. Структурная геоморфология Алтае-Саянской горной области. - [Structural geomorphology of Altai-Sayan mountain country] Новосибирск [Novosibirsk]. 168 pp. 